\title{
Propagation and structure of planar streamer fronts
}

\author{
Ute Ebert and Wim van Saarloos \\ Instituut-Lorentz, Universiteit Leiden, Postbus 9506, 2300 RA Leiden, The Netherlands \\ Christiane Caroli \\ Université Paris VII, GPS Tour 23, 2 place Jussieu, 75251 Paris Cedex 05, France
}

(Received 28 May 1996)

\begin{abstract}
Streamers are a mode of dielectric breakdown of a gas in a strong electric field: A sharp nonlinear ionization wave propagates into a nonionized gas, leaving a nonequilibrium plasma behind. The ionization avalanche in the tip of the wave is due to free electrons being accelerated in the strong field and ionizing the gas by impact. This chain reaction deeper in the wave is suppressed by the generated free charges screening the field. Simulations of streamers show two widely separated spatial scales: the width of the charged layer where the electron density gradients and the ionization rate are very large $[O(\mu \mathrm{m})]$, and the width of the electrically screened, finger-shaped, and ionized region $[O(\mathrm{~mm})]$. We thus recently have suggested analyzing first the properties of the charge-ionization layer on the inner scale on which it is almost planar, and then understanding the streamer shape on the outer scale as the motion of an effective interface, as is done in other examples of nonequilibrium pattern formation. The first step thus is the analysis of the inner dynamics of planar streamer fronts. For these, we resolve the long-standing question about what determines the front speed, by applying the modern insights of pattern formation to the streamer equations used in the recent simulations. These include field-driven impact ionization, electron drift and diffusion, and the Poisson equation for the electric field. First, in appropriately chosen dimensionless units only one parameter remains to characterize the gas, the dimensionless electron diffusion constant $D$; for typical gases under normal conditions $D \approx 0.1-0.3$. Then we determine essentially all relevant properties of planar streamer fronts. Technically, we identify the propagation of streamer fronts as an example of front propagation into unstable states. In terms of the marginal stability scenario we then find that the front approached asymptotically starting from any sufficiently localized initial condition (the "selected front") is the steepest uniformly translating front solution, which is physical and stable. Negatively charged fronts are selected by linear marginal stability, which allows us to derive their velocity analytically. Positively charged fronts can only propagate due to electron diffusion against the electric field; as a result their behavior is singular in the limit of $D \rightarrow 0$. For $D \leqq 1$, these fronts are selected by nonlinear marginal stability and we have to apply numerical methods for predicting the selected front velocity. For larger $D$, linear marginal stability applies and the velocity can be determined analytically. Numerical integrations of the temporal evolution of planar fronts out of localized initial conditions confirm all our analytical and numerical predictions for the selection. Finally, our general predictions for the selected front velocity and for the degree of ionization of the plasma are in semiquantitative agreement with recent numerical solutions of three-dimensional streamer propagation. This gives credence to our suggestion that the front analysis on the inner $(\mu \mathrm{m})$ scale yields the moving boundary conditions for a moving "streamer interface," whose pattern formation is governed by the evolution of the fields on the outer (mm) scale. [S1063-651X(96)09212-4]
\end{abstract}

PACS number(s): 47.54. $+\mathrm{r}, 52.80 . \mathrm{Mg}, 51.50 .+\mathrm{v}$

\section{INTRODUCTION}

Discharges are nonequilibrium ionization processes occurring in initially nonionized matter exposed to strong electric fields. Depending on the spatiotemporal characteristics of the electric field and on the ionization and charge transport properties of the medium, discharges can assume many different modes of appearance. In particular, in gases under approximately normal conditions one distinguishes phenomenologically between stationary modes such as arc, glow, or dark discharges and transient phenomena such as leaders, the initial stages of sparks, and streamers [1-6], which occur, e.g., in silent discharges [7]. The latter nonstationary discharges often form the initial state of a discharge that later on becomes stationary. We will focus here on an essential element of many transient discharge phenomena, the initial field-driven ionization wave.
The conceptually simplest problem of this kind has become known as the streamer problem in a nonattaching gas. It treats the dynamics of the free electrons and positive ions in a homogeneous gas at rest taking the following mechanisms into account: (i) impact ionization, the process in which a free electron accelerated in a strong local field ionizes a neutral molecule, generating a new free electron and a positive ion; (ii) drift and diffusion of charged particles, in particular of the electrons whose mobility is much larger than that of the ions; (iii) the coupling of the electric field to the charges through the Poisson equation of electrostatics.

Recent numerical simulations [8,9] of a basic model incorporating these physical ingredients for parameter values appropriate for nitrogen under normal conditions reveal that a streamer consists of a sharp nonlinear ionization front which propagates into a nonionized gas, leaving a weakly 
ionized nonequilibrium plasma behind. The underlying mechanism is that in the leading edge of the front the electrons are accelerated by the large imposed electric field; this causes the build-up of an electron avalanche due to impact ionization. The generated free charges eventually screen the field and thus suppress further ionization. It is the nonlinear balance between these two nonequilibrium processes, namely the ionization avalanche and the electric screening, which determines the dynamics of the ionization front and the state of the plasma behind it. In confined geometries, streamers usually have a nontrivial fingerlike shape, as is illustrated by the snapshots in Fig. 1 of streamer dynamics taken from the simulations of Vitello et al. [9]. As the sharpness of the electron density profiles in Fig. 1 illustrates, the "passive body" of the finger is separated from the external nonionized gas by a very narrow region - of width of order micrometers - in which essentially all the action is occurring. This width has to be compared to the size of the filament, which is of order millimeters. It is in this narrow layer that most of the ionization process is taking place. In this same region, there is a nonzero charge density, and consequently, also a very large electric field gradient. These features indicate that there are two different spatial scales in this process, an "inner" scale associated with the thickness of the zone where the ionization takes place, and an "outer" one where the spatial variations are set by the size of the finger and the external experimental geometry. It is precisely for these reasons that accurate simulations are extremely demanding and that they were accomplished only recently by Dhali and Williams [8] and by Vitello et al. [9]. (See also [10].)

Such a separation of scales is strongly reminiscent of what occurs in combustion fronts [11,12]. A combustion front is a narrow layer of thickness $l_{\text {in }}$ to which the combustion is essentially confined, while outside of it, the temperature field varies on a much longer scale $l_{\text {out }}$. Physically, such sharp combustion fronts occur in the limit when the chemical reaction rates involved in the combustion are very fast once a sufficient temperature is reached. It has been shown that, on the basis of an asymptotic expansion to lowest order in the small parameter $\varepsilon=l_{\text {in }} / l_{\text {out }}$ using matched asymptotic expansions $[13,14]$, the problem can be analyzed in terms of the propagation of an "effective interface." More specifically, one first solves the so-called inner problem of a locally almost planar reaction zone. This permits us to relate the temperature and chemical composition fields on both sides of the front (at distances $L$ such that $l_{\text {in }} \ll L \ll l_{\text {out }}$ ) and to determine the local front velocity as a function of local curvature and fields. On the scale of the remaining outer problem, these relations then play the role of boundary conditions and of a kinetic equation for the effective moving interface of zero thickness. Besides in combustion, the technique of asymptotic matching to obtain an effective interface description has also been applied to chemical waves [15], thermal plumes [16], and to phase field models of solidification $[17,18]$.

In spite of some important differences between combustion and streamer fronts as discussed in the Appendix, a similar approach appears possible for streamers. As discussed also in [19], building on such a reduced description of streamer dynamics appears very desirable, not only because
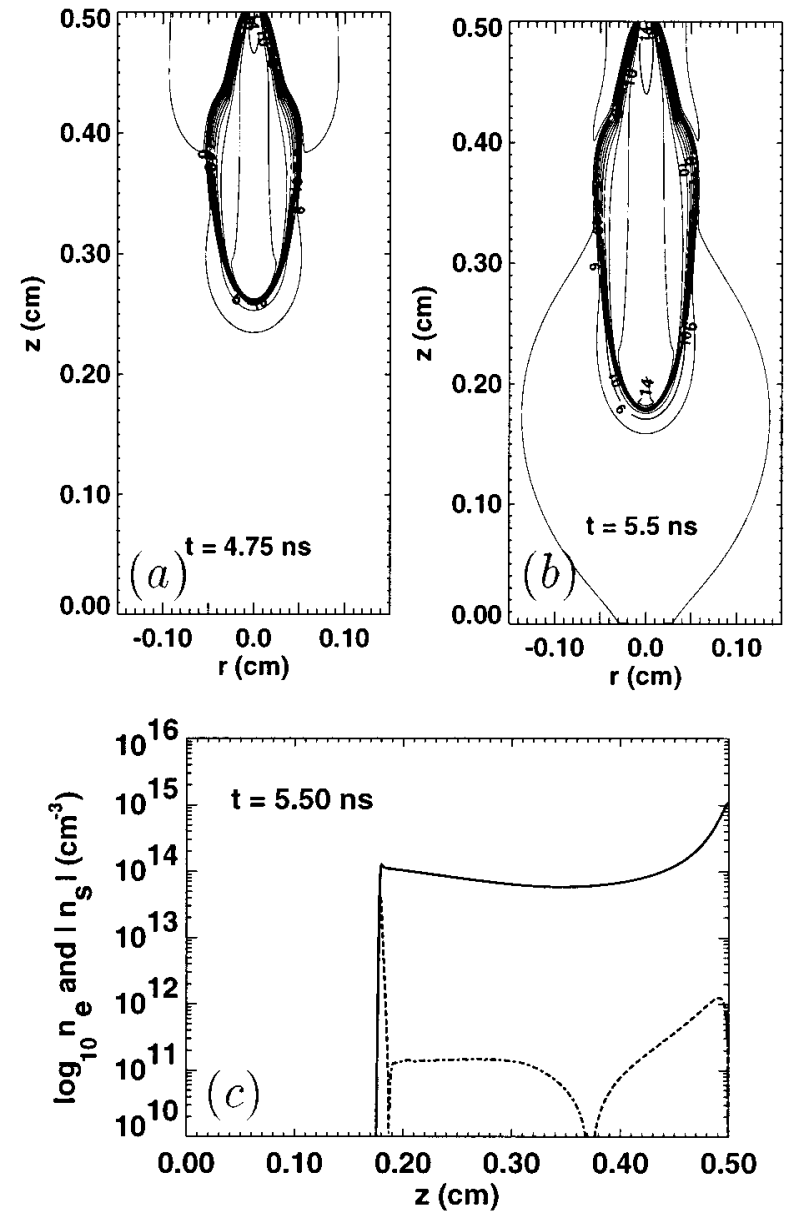

FIG. 1. Results of the numerical simulations of the full threedimensional streamer equations (2.1)-(2.6) of Vitello et al., reprinted from Figs. 1 and 10 in [9]. (a) Negative streamer propagating downwards towards the anode. Electrodes are planar and located at $z=0$ and $0.5 \mathrm{~cm}$; the voltage between the electrodes is 25 $\mathrm{kV}$, which in the absence of the streamer amounts to a constant electric field $|\mathcal{E}|=E_{0} / 4$. The system continues sidewards sufficiently far to make the lateral boundaries irrelevant. The streamer is assumed to be cylinder-symmetric. The dimensionless diffusion constant is $D=0.1$. Each line indicates an increase of $n_{e}$ by a factor 10; densities of $10^{3}-10^{14} \mathrm{~cm}^{-3}$ can be seen (initial background ionization: $1 \mathrm{~cm}^{-3}$ ). Shape at time $4.75 \mathrm{~ns}$ after an initial ionization seed was placed near the upper electrode. (b) Shape at time $5.5 \mathrm{~ns}$. (c) Logarithmic electron $n_{e}$ and total charge $n_{s}$ density along the symmetry axis of (b). Solid line, $n_{e}$; dot-dashed, $\left|n_{s}\right|$ for $n_{s}>0$; dotted, $\left|n_{s}\right|$ for $n_{s}<0$. Note the exponential increase of the densities on the $\mu \mathrm{m}$ scale within the front as well as the maximum of both densities in the rear part of the front. Courtesy of P. A. Vitello.

it might make numerical studies much easier, but also because it will allow us to draw upon the knowledge and methods which have been developed in the last decade in the field of interfacial pattern formation and dynamics [20]. The first step towards this goal is to determine the field dependence of the velocity and the ionization and charge profile of a planar front which propagates into the nonionized region. We thus analyze in this paper the inner problem for a planar streamer front. This allows us to reduce the problem to effectively one dimension. Our analysis clearly identifies the problem of streamer front propagation as an example of front propaga- 
tion into unstable states. Physically, the instability of the nonionized gas against charge fluctuations can be traced back to the fact that any small electron density gets amplified by the impact ionization. As is standard for front propagation into unstable states [21-25], we find that the onedimensional streamer equations exhibit a one-parameter family of uniformly translating front solutions, parametrized by their velocity. As usual [21-25], the question is then to decide which of these front solutions is the dynamically selected one, i.e., is the one reached at long times after a localized ionized region has been created by some initial ionization event. The existing knowledge of front propagation into unstable states $[22,23]$ provides us with an educated guess for the selected velocity, which we confirm with the help of numerical studies. Taken together, our results provide an essentially complete solution of the inner problem of planar streamer fronts.

In itself, the idea to analyze the planar fronts of a streamer model is not new - we refer to [26-29] for earlier work. Apart from the fact that the authors from the 1970s [26-28] investigate different models, which are more inspired by equilibrium concepts (e.g., the ionization behind the front is determined by thermal ionization, where the electron temperature is raised by application of strong electric fields), our work casts new light on this old problem from two different angles.

First it was empirically noted that the standard approach to analyze uniformly translating fronts failed to determine a unique propagation velocity, given the field and the gas parameters. Turcotte and Ong [26] clearly state this failure of their theory (this "great defect' of their theory is recalled in Fowler's reviews [28]) and suggest that a unique solution might be determined by a dynamical stability analysis. Albright and Tidman [27] then perform such a stability analysis, but not in a systematic way, and they draw incorrect conclusions. D'yakonov and Kachorovskii [29] also find the indeterminacy of the speed of uniformly translating planar fronts, now for an approximated version of our model, and propose to solve this by using the tip radius of the streamer finger as an extra length scale, which, however, they cannot determine. We, in contrast, trace the indeterminacy of the velocity from the analysis of uniformly translating streamer front solutions to the fact that this is an example of front propagation into unstable states. Applying the concepts explained above, we solve the selection problem for planar fronts without additional assumptions or approximations. We argue that a particular front solution out of a whole family of dynamically stable solutions is selected, because it is the only one compatible with the initial condition of a localized ionization seed.

Second, this result is the first ingredient for studying the formation of patterns, in particular of the tip radius - we do not attempt to model global features of the pattern formation with our planar front analysis. Our approach thus is very different in spirit from the earlier investigations: As also stressed in [19], in an effective interface description based on a matched asymptotic expansion, the results of weakly curved, almost planar fronts are essentially used locally everywhere in the interface region: They enter the analysis on the outer scale as boundary conditions at the moving interface. It is on this outer scale that pattern formation problems like the size, velocity, and shape of the streamer should be analyzed. Once our results on planar fronts will be extended to weakly curved fronts, all the necessary ingredients to tackle these questions appear to be available.

The main results of our present analysis of the streamer equations used in the simulations $[8,9]$ can be summarized as follows:

(a) Dimensional analysis shows that in dimensionless units, a single parameter remains to characterize the gas, the dimensionless electron diffusion coefficient $D$ characteristic of the gas [see Eq. (2.10)]. For gases under normal conditions, $D$ is small, of order $0.1-0.3$.

(b) The length scale set by the electron impact ionization coefficient [the coefficient $\alpha_{0}^{-1}$ in Eq. (2.5)] is on the order of micrometers for nitrogen. For $D \leqslant 1$ the thickness $l_{\text {in }}$ of the charged layer is on the order of this same ionization length for negatively charged streamer fronts (NSF) [30]. Given that typical streamer diameters found in the simulations are of the order of $1 \mathrm{~mm}, \varepsilon=l_{\text {in }} / l_{\text {out }}$ is at most of order $10^{-2}$; this justifies an effective interface description of streamer dynamics.

(c) We find that electron diffusion acts as a singular perturbation for positively charged streamer fronts (PSF): without diffusion, such fronts can not propagate, but with any nonzero $D$, they do. As a result, the behavior is singular in the limit $D \rightarrow 0$ : for $D=O(1)$, the thickness $l_{\text {in }}$ is again of order of the ionization length, but for $D \rightarrow 0$ the electron density and its gradients diverge due to the appearance of another smaller length scale (of order $D / \alpha_{0}$ ).

(d) The electron density generated by the propagating front is again basically set by dimensional analysis for NSF. We calculate for $D \leqslant 1.5$ the dependence of the dimensionless electron density $\sigma^{-}$behind the front on the electric field $E^{+}$far ahead of our planar front. Our results compare favorably with those extracted from the simulations [9], according to the prescriptions of the theory of matched asymptotic expansions $[13,14]$. Namely, $E^{+}$is not the field value at the electrode position, but the value obtained by extrapolating the slowly varying outer field to the front position. We also calculate the full $D$ and $E^{+}$dependence of the electron density $\sigma^{-}$behind the front of PSF for $D \leqq 1.5$.

(e) The dynamically relevant ("selected") front velocity $v_{f}$ is a unique function of $E^{+}$and $D$. The analysis confirms the strong asymmetry between NSF and PSF also found in the simulations $[8,9]$ for fronts propagating into an essentially nonionized region. The asymmetry is stronger the smaller $D$ is and disappears for $D \gg 1$.

(f) For NSF, $v_{f}$ is given by the so-called linear marginal stability velocity $v^{*}[22]$ — see Eq. (5.3) below. For parameter values used in the simulations, we find that $v_{f}$ is typically 30-40\% higher than the electron drift velocity just in front of the streamer head, which agrees semiquantitatively with the findings of Vitello et al. [9].

(g) We find that PSF propagate for any nonzero value of the dimensionless electron diffusion coefficient $D$. Due to the singular behavior as $D \rightarrow 0$, we find that fronts propagate with a unique velocity $v^{\dagger}$ predicted by the so-called nonlinear marginal stability mechanism [23] for small $D$. For the Townsend expression used in the simulations [8,9], this happens below a well-defined field-dependent value of $D$ of order unity (see Fig. 3). Above this threshold value, PSF 
propagate with the linear marginal stability value $v^{*}$.

In this paper, our main focus will be on those results that are of greatest interest from the point of view of understanding the generation of low temperature plasmas by the streamer mechanism. We note, however, that the equations for planar streamer fronts [Eqs. (3.11) and (3.12) below] appear to be of interest in their own right. As will be discussed briefly in Sec. V, our streamers have several features in common with the celebrated nonlinear diffusion equation studied in mathematics $[31,32]$ since the early work of Kolmogorov et al. [33] and Fisher [34]; at the same time, however, they are sufficiently more complicated that they appear to present new challenges from a mathematical point of view.

This paper is organized as follows. In Sec. II we introduce the basic equations for streamer formation, and perform a dimensional analysis for the inner problem of streamer fronts. In Sec. III, we discuss the stability of the basic homogeneous states of interest, the homogeneous nonionized state, and the homogeneous weakly ionized state. We also discuss the physical mechanism of streamer formation and the proper initial and boundary conditions to study these in the case of planar fronts, which allow us to simplify the equations describing planar front dynamics. In Sec. IV we demonstrate that there exists a one-parameter family of uniformly translating fronts characterized by a continuous range of front velocities $v$. We also briefly show how in the case $D=0$, the equations for uniformly translating fronts can be solved analytically. These solutions, which turn out to be useful as a small- $D$ approximation for NSF, yield an explicit expression for the electron density $\sigma^{-}$behind the NSF in terms of the field $E^{+}$just ahead of it. This is followed by an analysis of the general case $D \neq 0$; then the equations cannot be solved analytically, but we demonstrate that there still is a one-parameter family of uniformly translating front solutions. For PSF, we show that the limit $D \rightarrow 0$ is singular; we discuss this limit in detail and show that it accounts for the strong asymmetry between PSF and NSF for realistic values of $D$. In Sec. V we then summarize some of the main results [21-25] concerning the so-called selection problem, the question of which particular front solution from the family is reached asymptotically for large times for a large class of initial conditions. Application of these concepts allows us to predict the shape and velocity of the dynamically relevant front solution (the selected front) and the value of the electron density generated behind it. This yields the various selection results for NSF and for PSF, summarized in points (c) - (g) above, and leads us to predict that the behavior of PSF in the limit $D \rightarrow 0$ is singular. In Sec. VI we present numerical simulations of the full partial differential equations for planar streamer dynamics; starting from various initial conditions, we illustrate that in all cases we have studied, the long time dynamics of the system is characterized by a NSF and a PSF whose behavior is in full agreement with our predictions. In the concluding section we finally reflect on our results and on the future steps to be taken to arrive at an effective interface description of streamer dynamics. In an Appendix we discuss differences and similarities between combustion and streamer fronts.

\section{MODELING AND DIMENSIONAL ANALYSIS}

\section{A. The minimal streamer model}

For simulating the dynamical development of streamers out of a macroscopic initial ionization seed in a so-called nonattaching gas like $\mathrm{N}_{2}$ under normal conditions, Dhali and Williams [8], and Vitello et al. [9] use the following set of deterministic continuum equations for the electron density $n_{e}$, the ion density $n_{+}$and the electric field $\mathcal{E}$ : balance equations for electrons and ions,

$$
\begin{gathered}
\partial_{t} n_{e}+\nabla_{\mathbf{R}} \cdot \mathbf{j}_{e}=\text { source } \\
\partial_{t} n_{+}+\nabla_{\mathbf{R}} \cdot \mathbf{j}_{+}=\text {source }
\end{gathered}
$$

where the fact that the two source terms are the same is due to charge conservation in an ionization event; the Poisson equation,

$$
\boldsymbol{\nabla}_{\mathbf{R}} \cdot \mathcal{E}=\frac{\mathrm{e}}{\varepsilon_{0}}\left(n_{+}-n_{e}\right)
$$

and the approximate phenomenological expressions

$$
\begin{gathered}
\mathbf{j}_{e}=-n_{e} \mu_{e} \mathcal{E}-D_{e} \nabla_{\mathbf{R}} n_{e}, \\
\mathbf{j}_{+}=\mathbf{0} \\
\text { source }=\left|n_{e} \mu_{e} \mathcal{E}\right| \alpha_{0} e^{-E_{0} /|\mathcal{E}|} .
\end{gathered}
$$

Apart from the fact that we will allow for a slight generalization of Eq. (2.6), these are the equations that we will investigate analytically below.

In these equations, $\mathbf{j}_{e}$ and $\mathbf{j}_{+}$are the particle current densities of electrons and positive ions, and $\mathrm{e}$ is the absolute value of the electron charge. The (dimensional) spatial coordinates are denoted by $\mathbf{R}$, and $\boldsymbol{\nabla}_{\mathbf{R}}$ is the gradient with respect to these coordinates. The use of only Poisson's law of electrostatics, Eq. (2.3), means that all magnetic fields, as well as terms in the Maxwell equations associated with time dependences of the fields, are neglected [35].

The electron particle current density $\mathbf{j}_{e}$ is approximated in Eq. (2.4) as the sum of a drift and a diffusion term. Note that this diffusion approximation implies that the electron mean free path must be small with respect to the scale of variation $l_{\text {in }}$ of the electric field. This condition is just about satisfied for the parameter values taken for $N_{2}$ in the simulations, except possibly at the highest field values (see also the discussion in Sec. VII). The electron drift velocity is taken to be linear in the field $\mathcal{E}$, with $\mu_{e}$ the (positive) electron mobility. The electron diffusion coefficient $D_{e}$ and the mobility $\mu_{e}$ are treated here as independent coefficients, since they effectively depend on the field strength [3] (only in the low-field limit are they related by the Einstein relation). More generally, the diffusion coefficient should be replaced by a diffusion tensor, which is diagonal in a reference frame with one axis along the electric field. Its longitudinal component, the only relevant one for planar fronts perpendicular to $\mathcal{E}$, is somewhat smaller than the transverse one. Since we will see that $N_{2}$ reaches a typical degree of ionization of only $10^{-5}$, density fluctuations of the nonionized gas can be neglected and the mean free path of the electrons and therefore $\mu_{e}$ and $D_{e}$ can be taken as independent of the degree of ionization.

The ionic current is neglected according to Eq. (2.5), since the mobility of ions is at least two orders of magnitude smaller than that of the electrons [8]. In particular, for the 
analysis of the inner scale, that we will perform in the present paper, $\mathbf{j}_{+}$is negligible.

The source (2.6) finally accounts for the creation of free charges by impact ionization. If the product of electric field $\mathcal{E}$ and electronic mean free path $l_{\mathrm{mfp}}$ is large enough, free electrons can gain sufficient kinetic energy to ionize neutral molecules. Accordingly there is a threshold field $|\mathcal{E}|=E_{0}$ $\propto l_{\text {mfp }}^{-1}$ For $|\mathcal{E}| \gtrsim E_{0}$ the probability that a scattering event carries at least the ionization energy is large. The effective ionization cross-section $\sigma_{c s}(|\mathcal{E}|)$ then essentially saturates, while for $|\mathcal{E}| \ll E_{0}$ the ionization rate per scattering event is largely suppressed. The source term is given by the ionization rate, which can be calculated as the product of the drift current of free electrons $\left|n_{e} \mu_{e} \mathcal{E}\right|$ times the target particle density $n_{n}$ of the neutral gas times the effective ionization cross section $\sigma_{c s}(|\mathcal{E}|)$. Commonly, a phenomenological ionization coefficient $\alpha(|\mathcal{E}|)=n_{n} \sigma_{c s}(|\mathcal{E}|)$ is used, (which clearly has dimension of inverse length,) whose field threshold behavior in the Townsend approximation $\alpha(|\mathcal{E}|)$ $=\alpha_{0} \exp \left(-E_{0} /|\mathcal{E}|\right)[3]$ is expressed by Eq. (2.6). As discussed by Raizer [3], in the approximation that every collision is ionizing, if the electron carries an energy larger than the ionization energy $I$, we have

$$
\alpha_{0} \approx l_{\mathrm{mfp}}^{-1}, \quad \text { and } E_{0} \approx I /\left(\mathrm{e} l_{\mathrm{mfp}}\right) .
$$

Since in much of our analysis the specific form of $\alpha(|\mathcal{E}|)$ is not needed, we will use a slightly more general formulation in Eq. (2.11) below.

In the source term, ionization due to the photons also created in recombination or scattering events is neglected. This is motivated by the ionization cross sections due to photons being much smaller than those due to electrons. Note that, if photoionization is taken into account, the dynamical equations become nonlocal.

No sink term needs to be included for the analysis of the inner problem, since the recombination length at a degree of ionization of order $10^{-5}$ that we will derive below is very large as compared with the front width $l_{\text {in }}$. (For this reason, the inner problem is the same for streamers and leaders [3]: the difference between these discharge modes, which consists in the fact that recombination is negligible in the plasma body of leaders, would come into play only when solving, at a later stage, the outer problem.) The fact that the degree of ionization remains small is also the reason that saturation effects are neglected in Eq. (2.6).

In contrast to the situation in $\mathrm{N}_{2}$, which is described by our model equations, in attaching gases like $\mathrm{O}_{2}$, a third charged species plays a role, namely, negative ions formed by a neutral molecule catching a free electron. For a description of the physics of such attaching gases and simulations thereof, see, e.g., [36].

The equations above are deterministic. Thermal fluctuations in fact can be neglected, since even an unphysically small ionization energy of $3 \mathrm{eV}$ leads to a Boltzmann factor of $10^{-52}$ at room temperature. Also other stochastic effects are not accounted for in the simulations we compare to. We further discuss possible stochastic effects in the experiments in the Conclusion.

Finally, the dynamical system (2.1)-(2.6) must be complemented by the following. (i) Boundary conditions: as will be discussed in detail in Sec. III, for the problem of front propagation, these are specified by the value $E^{+}$of the electric field far ahead of the front, where the total charge density vanishes.

(ii) Initial conditions: we ignore the details of the plasma nucleation event (e.g., triggering by radiation from an external source), and assume that at $t=0$ a small well-localized ionization seed is present. The precise meaning, for our problem, of "well-localized" will be made clear in Sec. V.

\section{B. Dimensional analysis}

In order to identify the physical scales and intrinsic parameters of our problem, we reduce Eqs. (2.1)-(2.6) to a dimensionless form. The most natural scale of length and electric field are the ionization length $l_{\text {ion }}=\alpha_{0}^{-1}$ and the threshold field $E_{0}$ of the ionization rate (2.6). The velocity scale is then the electron drift velocity at this field strength, $v_{0}=\mu_{e} E_{0}$, leading to a time unit $t_{0}=\left(\alpha_{0} \mu_{e} E_{0}\right)^{-1}$, and a charge unit $q_{0}=\varepsilon_{0} \alpha_{0} E_{0}$.

For concreteness, we list here the values of these quantities for $\mathrm{N}_{2}$ at normal pressure, used in the simulations $[8,9]$

$$
\begin{gathered}
\alpha_{0}^{-1} \approx 2.3 \mu \mathrm{m}, \quad v_{0} \approx 7.56 \times 10^{7} \mathrm{~cm} / \mathrm{s}, \\
t_{0} \approx 3 \cdot 10^{-12} \mathrm{~s}, \quad q_{0} \approx 4.7 \times 10^{14} \mathrm{e} / \mathrm{cm}^{3} \\
E_{0} \approx 200 \mathrm{kV} / \mathrm{cm}, \quad \mu_{e} \approx 380 \mathrm{~cm}^{2} / \mathrm{Vs} .
\end{gathered}
$$

We now introduce dimensionless quantities by defining

$$
\begin{array}{cc}
\mathbf{r}=\mathbf{R} \alpha_{0}, & \boldsymbol{\tau}=t / t_{0}, \\
q=\left(n_{+}-n_{e}\right) \mathrm{e} / q_{0}, & \sigma=n_{e} \mathrm{e} / q_{0}, \\
\mathbf{j}=-\mathbf{j}_{e} \mathrm{e} /\left(q_{0} v_{0}\right), & \mathbf{E}=\boldsymbol{E} / E_{0} .
\end{array}
$$

Note that with our definition, $\mathbf{j}$ now plays the role of a dimensionless charge current. If we furthermore introduce the dimensionless diffusion coefficient $D$ as

$$
D=D_{e} \alpha_{0} / \mu_{e} E_{0},
$$

we obtain what we call the streamer equations

$$
\begin{aligned}
\partial_{\tau} \sigma-\boldsymbol{\nabla} \cdot \mathbf{j} & =\sigma f(|\mathbf{E}|), \\
\partial_{\tau} q+\boldsymbol{\nabla} \cdot \mathbf{j} & =0, \\
q & =\boldsymbol{\nabla} \cdot \mathbf{E}, \\
\mathbf{j} & =\sigma \mathbf{E}+D \boldsymbol{\nabla} \sigma,
\end{aligned}
$$

where $\boldsymbol{\nabla}$ denotes the gradient with respect to the dimensionless coordinate $\mathbf{r}$, and where the "ionization function"

$$
f(|\mathbf{E}|)=|\mathbf{E}| \alpha(|\mathbf{E}|) / \alpha_{0}
$$

is assumed to vanish at zero field. Townsend's expression (2.6) yields

$$
f_{T}(|\mathbf{E}|)=|\mathbf{E}| \exp (-1 /|\mathbf{E}|) .
$$

In general, we will treat an ionization function with the properties [37] 


$$
f(0)=0=f^{\prime}(0) \text { and } f^{\prime}(|\mathbf{E}|) \geqslant 0 \text { for all }|\mathbf{E}| \text {. }
$$

The dimensionless equations (2.11)-(2.14) now depend on only one internal parameter, the dimensionless diffusion coefficient $D$. For the values used in $[8,9]$ for $\mathrm{N}_{2}$ under normal conditions, $D \approx 0.1$, while according to the data given by Raizer [3], for $\mathrm{Ne}$ and $\mathrm{Ar}, D \approx 0.3$. We believe that typical values are generally in the range $0.1-0.3$, since in the approximation (2.7), $\alpha_{0} / E_{0} \approx I / \mathrm{e}$ and since the ratio $D_{e} / \mu_{e}$ appears to be commonly of the order of volts for large fields, while $I$ is typically of the order of several electron volts.

We are now able, solely on the basis of the dimensional analysis above, to make a first semiquantitative prediction about streamers. We will in practice be interested in external fields $E^{+}=O(1)$ (for $E^{+} \ll 1$ and $\alpha_{0}^{-1}$ on the order of micrometers, the electron avalanche process becomes much too ineffective for streamer fronts to develop at reasonably small distances; also our scale separation approach discussed in the Introduction might break down). We can therefore expect that, for $D$ values $\lesssim 1$, as is the case for $\mathrm{N}_{2}$, front widths will

be of order $\alpha_{0}^{-1}$, and that in addition the reduced electron density $\sigma^{-}$far behind the front on the inner scale will be of order unity as well. This leads one to expect electron densities in the streamer body on the order of $10^{14} \mathrm{~cm}^{-3}$, in agreement with numerical findings.

\section{HOMOGENEOUS SOLUTIONS AND THE CONCEPT OF FRONTS}

\section{A. Homogeneous states and their stability}

The first task, when studying in general the propagation of a front, is to identify the nature and stability of the states that the front connects. We expect the invading state, here the ionized one created by the front, to be stable [38], while the invaded state can in general either be metastable or unstable. Physically, we of course expect the nonionized state to be unstable in a nonvanishing field in the present model. (In an attaching gas forming also negative ions, it is conceivable that the nonionized state is metastable for not too strong fields.)

Equations (2.11)-(2.14) immediately yield that stationary homogeneous states simply are solutions of

$$
\sigma f(|\mathbf{E}|)=0 .
$$

So, these stationary states decompose into two families, as follows.

(i) Nonionized states, with $\sigma=0, \mathbf{E}$ arbitrary: Since the density of free electrons vanishes, no ionization can occur, whatever the value of $\mathbf{E}$ is. If also the density of ions vanishes, $\boldsymbol{\nabla} \cdot \mathbf{E}=\mathbf{0}$. Since these states correspond to the physical situation far ahead of the front, we label them $(+)$. Moreover, since we will need in particular the case in which the field ahead of the front is constant, we take $\mathbf{E}^{+}=$const.

(ii) Completely screened states, labeled ( - ), with $\mathbf{E}=\mathbf{0}$, $\sigma^{-}$arbitrary [39]: Whatever the electron density, for $\mathbf{E}=\mathbf{0}$ impact ionization does not occur and thermal energy is much too small to permit ionization.
Since the steady states we consider as well as the equations of motion are translation invariant in space and time, the eigenstates of the linear perturbations are Fourier modes of the form

$$
\left(\begin{array}{c}
\delta \sigma(\mathbf{r}, t) \\
\delta \mathbf{E}(\mathbf{r}, t)
\end{array}\right)=\left(\begin{array}{l}
\sigma_{1} \\
\mathbf{E}_{1}
\end{array}\right) \exp (i \mathbf{k} \cdot \mathbf{r}+\omega \tau) .
$$

We first investigate the linear stability of the nonionized state $\sigma^{+}=0$. Upon linearizing the equations about the zeroth order values $\left(\sigma^{+}=0, \mathbf{E}^{+}\right)$, we find two branches of modes.

(a) The first, trivial branch is a zero mode $(\omega=0)$, with $\sigma_{1}=0$, expressing that the electron density remains zero. This zero mode accounts for the degeneracy of the nonionized states, i.e., for the fact that there exists a $(+)$ stationary state for each value of $\mathbf{E}^{+}$. (For $\mathbf{E}^{+} \neq$const, these zero modes express the degeneracy of all steady states with $q^{+}=\boldsymbol{\nabla} \cdot \mathbf{E}^{+}$ for any ion density $q^{+}$as long as the electron density $\sigma^{+}$ vanishes.)

(b) The second branch of perturbations is associated with fluctuations carrying a finite electron charge; its dispersion relation is

$$
\omega^{+}=i \mathbf{k} \cdot \mathbf{E}^{+}+f\left(\left|\mathbf{E}^{+}\right|\right)-D k^{2},
$$

with $i \omega^{+} \mathbf{k} \cdot \mathbf{E}_{1}=\left[f\left(\left|\mathbf{E}^{+}\right|\right)-\omega^{+}\right] \sigma_{1}$. The first term on the right-hand side of Eq. (3.3) simply expresses the fact that the electrons drift, to first order, in the electric field $\mathbf{E}^{+}$with velocity $\left(-\mathbf{E}^{+}\right)$. The real part $\operatorname{Re} \omega^{+}$, the sign of which determines whether fluctuations decay or are amplified, contains a destabilizing term, expressing that any small electron density fluctuation is amplified at rate $f$, and a stabilizing term, due to the diffusive spreading of electron charges. For $k^{2}<f\left(\left|\mathbf{E}^{+}\right|\right) / D, \operatorname{Re} \omega^{+}>0$ : nonionized states are unstable against long-wavelength perturbations.

We note that the single Fourier eigenmodes (3.2) violate individually the physical constraint that $\sigma$ be positive everywhere. But Eq. (3.3) also determines the time evolution of physically allowed fluctuations (wave packets) that are superpositions of these eigenmodes. For example, one easily deduces from it Lozanski's expression [40] for the time evolution of a Gaussian-shaped small electron density with arbitrary constants $c_{1}, c_{2}>0$,

$$
\delta \sigma(\mathbf{r}, \tau)=c_{1} e^{f\left(\left|\mathbf{E}^{+}\right|\right) \tau} \frac{e^{-\left(\mathbf{r}+\mathbf{E}^{+} \tau\right)^{2} /\left(c_{2}+4 D \tau\right)}}{\left(c_{2}+4 D \tau\right)^{3 / 2}},
$$

as long as linearization around the nonionized state holds. As expected, the center of the spreading packet drifts with velocity $-\mathbf{E}^{+}$, while the total number of electrons it contains is amplified at rate $f$ and the wave packet stays Gaussian, with time-dependent width $c_{2}+4 D \tau$. Such ionization modes derived by linearizing around the nonionized state are known as electron or ionization avalanches in the gas discharge literature.

We now perform the same linear stability analysis for the completely screened states $\left(\sigma^{-}=\right.$const, $\left.\mathbf{E}^{-}=\mathbf{0}\right)$. The fact that $f^{\prime}(0)=0$ from Eq. (2.17) assures that the linear perturbations are not affected by ionization; the dynamics thus evolves with conserved particle densities.

Again, due to the existence of a continuous family of screened stationary states, parametrized by $\sigma^{-}$, the spectrum 
contains a branch of $\omega=0$ modes. For the nontrivial branch, the dispersion relation is given by

$$
\omega^{-}=-\sigma^{-}-D k^{2},
$$

while the eigendirection of such a perturbation is given by

$$
\sigma_{1}+i \mathbf{k} \cdot \mathbf{E}_{1}=\sigma_{1}+q_{1}=0 .
$$

Since $\left(\sigma_{1}+q_{1}\right)$ is the dimensionless ion density of the linear mode, Eq. (3.6) simply expresses the fact that ions are completely immobile in our model.

Equation (3.5) expresses the fact that the completely screened $(-)$ states are stable, the decay of perturbations being due to the added stabilizing effects of overdamped plasmons $\left(-\sigma^{-}\right)$and electron diffusion. The $\mathbf{k} \rightarrow \mathbf{0}$ limit of the plasmon mode leads to dielectric screening [41].

\section{B. The mechanism of front creation}

Let us now investigate the dynamical evolution of an initial state in which the electron and ion densities vanish everywhere except in a small localized region. An example of such localized initial conditions is an initially Gaussian electron density, as in the simulations $[8,9]$ — under what circumstances initial conditions are sufficiently localized will become clear later. As long as the electron and ion densities are small enough, we can neglect in linear approximation the changes in the field as we did above when linearizing about the nonionized state. As a result, both densities will grow due to impact ionization. If this were the only mechanism, the space charge would remain unchanged and the ionization would continue indefinitely. However, the electrons are mobile, and at the same time they start to drift in the direction opposite to the electric field $\mathbf{E}$. If we neglect for the moment the diffusion, this drift has two effects. First of all, the electrons start to drift in the direction of the anode. Impact ionization then starts in previously nonionized regions as well, so the ionized region expands towards the anode. Second, as the electrons drift while the ions stay put (on the fast time scale), a charge separation occurs which tends to suppress the field strength in the ionized region. When the size of the initial perturbation and/or the time during which the avalanche has built up are large enough, the screening of the field becomes almost complete in the ionized region so that ionization stops there. The behavior in this region can be described by linearizing around the screened state as was done above. After an electrically screened body of the ionized region has developed, the initial ionization avalanche is said to have developed into a streamer. Thus streamer fronts are strongly nonlinear and determined by two competing mechanisms, which dynamically balance each other: the ionization process which is strongest at the leading edge and the screening of the field due to the free charges which increases towards the rear end of the front. This balance also explains our finding that the ionization length and the screening length in the plasma behind the NSF are of the same order of magnitude for field values that are not too small. Technically speaking, the challenge in constructing the full front is to connect the two regimes linearized about the homogeneous states in an appropriate way through the nonlinear regime of the front.
In the above discussion, we have neglected electron diffusion. In this case the NSF propagates towards the anode with a velocity that is at least the drift velocity of the electrons in the local electric field. The PSF, in contrast, is moving in the direction opposite to the drift of the only mobile species, the electrons. Its space charge is formed by the ions staying put, while the electrons are drawn into the ionized body. Propagation of a PSF is therefore only possible if the electron diffusion current overcompensates the drift current. This in turn implies that if the diffusion coefficient $D$ is small, electron density gradients must be extremely steep. From this discussion it already becomes evident - and we will derive this below - that for an NSF, diffusion is a small correction for $D \ll 1$, since drift and diffusion currents are acting in parallel directions. In PSF, however, diffusion has to overcome the drift, and as a result in this case the limit of vanishing diffusion is very singular. We will see in Sec. IV that this manifests itself through the emergence of a new inner length scale $D / \alpha_{0}=D_{e} /\left(\mu_{e} E_{0}\right)$, the diffusion length associated with the electron drift velocity.

Of course, a charged front only screens the normal component of the electric field. This is why electric screening is efficient in the head of the streamer, while the field penetrates in the body of a single streamer in the simulations $[8,9]$. Our planar front analysis thus serves as a first approximation for the mechanisms in the moving tip of the streamer finger.

\section{The one-dimensional streamer equations}

Let us now restrict our analysis to the case of plane fronts perpendicular to a constant electric field. Of course, in practice planar streamer fronts will be unstable to deformations along the front (very much like in the Mullins-Sekerka instability in crystal growth [20]), but as explained in the Introduction, the planar front analysis is a first step towards understanding the dynamics on the inner length scale $\alpha_{0}^{-1}$ and time scale $t_{0}$. As such, it is the first basic ingredient for deriving an effective interface model on scales $\gg \alpha_{0}^{-1}$.

We choose the $x$ axis as parallel to the field and perpendicular to the planar front so that $\mathbf{E}=E \hat{\mathbf{x}}$ and $\nabla=\hat{\mathbf{x}} \partial_{x}$. From the point of view of matched asymptotic expansions, the electric field in the nonionized region before the front will vary adiabatically slowly on the "inner" time scale $\tau$ of the front, the time scale on which the front propagates over a distance comparable to its width, because the length scales of the outer problem determining the changes of $E$ are assumed to be much larger than the inner scale $\alpha_{0}^{-1}$. For our study of the inner problem, we thus take the asymptotic field value $\mathbf{E}^{+}$in the unionized region constant in time. Furthermore, we will use the convention that the nonionized initial state into which the front propagates is at the right towards large positive values of $x$, so that there

$$
\sigma \rightarrow \sigma^{+}=0, \quad q \rightarrow q^{+}=0, \quad E \rightarrow E^{+}, \partial_{\tau} E^{+}=0 \quad \text { for } x \rightarrow+\infty,
$$

which motivates now the use of the superscript + . We emphasize again that " $x \rightarrow+\infty$ " should be interpreted on the length scale $\alpha_{0}^{-1}$ of the inner problem in the sense of matched asymptotic expansions [13,14]. Far behind the 
front, i.e., for $x \rightarrow-\infty$, the discussion of Sec. II leads us to expect a homogeneous stable state

$$
\sigma \rightarrow \sigma^{-} \neq 0, q \rightarrow q^{-}=0, E \rightarrow 0 \text { for } x \rightarrow-\infty .
$$

Which value $\sigma^{-}$will be dynamically selected and what the corresponding front velocity and profile are, for a given fixed value of the electric field $E^{+}$before the front, is the selection problem we aim to solve.

The boundary condition (3.7) allows an important simplification of the equations in one dimension: If we insert Eq. (2.13) into (2.12) and integrate, we obtain

$$
\partial_{\tau} E+j=h(\tau)
$$

where $h(\tau)$ is an arbitrary function of time which is constant in space. In view of the boundary condition $(3.7), h(\tau)$ vanishes at $x \rightarrow \infty$ and thus everywhere. For planar fronts, the model Eqs. (2.11)-(2.14) then reduce to

$$
\begin{aligned}
& \partial_{\tau} \sigma=\partial_{x}(\sigma E)+D \partial_{x}^{2} \sigma+\sigma f(|E|), \\
& \partial_{\tau} E=-\sigma E-D \partial_{x} \sigma
\end{aligned}
$$

with space charge and electric current determined by

$$
q=\partial_{x} E \quad \text { and } j=\sigma E+D \partial_{x} \sigma .
$$

We will refer to Eqs. (3.10) and (3.11) as to the onedimensional streamer equations. They are the basic equations of this paper, on which the rest of our analysis will be based.

Equation (3.11) implies that the field decays behind the front, if no strong density gradients act against it. As we shall see later when we will discuss our simulation results in Sec. VI, such strong density gradients often occur during the transient regime before a PSF emerges. Once, however, a front has approached an approximately uniformly translating state, the electron density $\sigma^{-}$behind the front is almost homogeneous and the field behind the front then decays to zero on a time scale $1 / \sigma^{-}$according to Eq. (3.11). Note that the local decay of the field for any nonzero electron density is due to electrodynamics of conserved quantities that continues also after the impact ionization has been suppressed.

We finally note that in the limit where the diffusion is small $(D \ll 1)$, it is easy to identify the crossover time from the linear avalanche regime to that of streamer propagation in the case that the initial electron density is small and nonzero only in a very narrow localized region. As explained in the beginning of this section, in the avalanche regime we can neglect the changes in the background field $E^{+}$due to the build-up of the charges. The evolution of the electron density is then described by the linearized version of Eq. (3.10), a linear equation with drift, diffusion, and growth. Hence, if the initial electron density is, e.g., Gaussian, the electron density will, according to Eq. (3.4), remain a Gaussian profile, whose maximum drifts with a velocity $|\mathbf{E}|$ in the direction opposite to the field and whose amplitude grows exponentially as $\exp \left(f\left(E^{+}\right) \tau\right)$. In other words, if the total initial electron charge is $N_{e}(0)=\int d x \sigma(x, 0)$, then the total number of electrons in this avalanche regime grows as $N_{e}(\tau)=N_{e}(0) \exp \left(f\left(E^{+}\right) \tau\right)$. Likewise, the total ion charge grows exponentially, but if both the diffusion constant and the width and amplitude of the initial perturbation are small, the electron drift will separate the negative electron charge and the positive ion charge almost completely. The crossover to the nonlinear streamer regime will therefore occur when the total charge in the positively and negatively charged regions is big enough that screening of the field becomes appreciable, i.e., at a time $\tau_{c}$ when

$$
N_{e}\left(\tau_{c}\right) \approx\left|E^{+}\right|, \quad \Rightarrow \tau_{c} \approx \frac{1}{f\left(E^{+}\right)} \ln \left[\left|E^{+}\right| / N_{e}(0)\right] .
$$

\section{UNIFORMLY TRANSLATING FRONT SOLUTIONS}

Above we already have introduced the idea that fronts asymptotically approach some shape, which is independent of the initial conditions. This is based on our experience [21-25] with other examples of front propagation into unstable states that the front will acquire some asymptotic shape and velocity in the long time limit, which will be the same ("universal") for a large class of "sufficiently localized" initial conditions that comprise most physically relevant initial states. This property is often referred to as the front selection problem. Our subsequent analysis will therefore follow the usual strategy in examples of this type: We will first show in this section that there generally is a oneparameter family of front solutions. In Sec. V we then summarize our present understanding of the front selection problem, and on the basis of this predict the properties of the selected streamer front. The numerical simulations that confirm our predictions are presented in Sec. VI.

Uniformly translating fronts with velocity $v$ are stationary in a coordinate system moving with velocity $v$. If we denote this comoving coordinate by $\xi=x-v \tau$, the partial differential equations (PDE's) (3.11) and (3.12) in this coordinate system become

$$
\begin{aligned}
& \left.\partial_{\tau} \sigma\right|_{\xi}=v \partial_{\xi} \sigma+\partial_{\xi}(\sigma E)+D \partial_{\xi}^{2} \sigma+\sigma f(|E|), \\
& \left.\partial_{\tau} E\right|_{\xi}=v \partial_{\xi} E-\sigma E-D \partial_{\xi} \sigma .
\end{aligned}
$$

A front translating uniformly with velocity $v$ in the fixed frame $x$ is stationary in this comoving frame, $\left.\partial_{\tau} \sigma\right|_{\xi}=0=\left.\partial_{\tau} E\right|_{\xi}$. As a result, the corresponding front profiles are solutions of the ordinary differential equations (ODE's). (We continue to use partial differential signs $\partial_{\xi}$ even though the uniformly translating solutions are functions of the variable $\xi$ only.)

$$
\begin{gathered}
D \partial_{\xi}^{2} \sigma+(v+E) \partial_{\xi} \sigma+\sigma \partial_{\xi} E+\sigma f(|E|)=0, \\
D \partial_{\xi} \sigma-v \partial_{\xi} E+\sigma E=0 .
\end{gathered}
$$

These equations are analyzed below. Both for $D=0$ and for $D \neq 0$, they admit solutions for a range of values of the velocity, so we are indeed faced with the question of front selection.

It is important to realize that not all the exact uniformly translating front solutions of these ODE's correspond to physically relevant solutions. In particular, any physical electron density $\sigma$ needs to be non-negative $(\sigma \geqslant 0)$, but as we shall see the set (4.2) admits PSF solutions where $\sigma$ goes 
negative. We expect these solutions to be unstable (in accord with the "nonlinear marginal stability" scenario [23]), and also not to be approachable from an initial condition with $\sigma \geqslant 0$. Hence they are neither dynamically nor physically relevant. Furthermore, note that in our model the ion density $q_{i}(=\rho+\sigma)$ can only increase due to impact ionization [Eqs. (2.11) and (2.11) imply $\left.\partial_{\tau} q_{i}=\sigma f(E) \geqslant 0\right]$. With our convention that the nonionized state is on the right, this implies that uniformly receding front solutions with $v \leqslant 0$ are unphysical. We will therefore call a uniformly translating front solution physical if

$$
v>0 \text { and } \sigma(\xi) \geqslant 0 \text { for all } \xi
$$

\section{A. $D=0$ Front solutions}

In contrast to the case $D \neq 0$, where we can derive properties of uniformly translating fronts only either qualitatively by discussing flows in phase space or quantitatively by numerical integration, Eqs. (4.2) for $D=0$ can be integrated explicitly. Doing so, we derive a simple explicit expression for the electron density $\sigma^{-}$behind the front in terms of the field $E^{+}$before the front; this analysis generalizes an earlier result of D'yakonov and Kachorovskii [29], and explicitly illustrates the existence of a family of uniformly translating solutions. For NSF, these results extend smoothly to the case $D \neq 0$ : The electron density $\sigma^{-}\left(E^{+}\right)$derived for $D=0$ will turn out to be a good approximation for $D \lesssim 1$, and the small overshoot of $\sigma$ at the rear end of the front visible in the three-dimensional simulations in Fig. 1(c), is also recovered for $D=0$. For PSF, on the other hand, we will see that $D$ acts as a singular perturbation, so that the class of $D=0$ PSF solutions that we derive here is not relevant for the PSF selection problem for $D \lesssim 1$.

The ODE's describing uniformly translating fronts for vanishing diffusion are found by putting $D=0$ in Eq. (4.2). These equations then become

$$
\begin{gathered}
\partial_{\xi}[(v+E) \sigma]=-\sigma f(|E|), \\
v \partial_{\xi} \ln |E|=\sigma .
\end{gathered}
$$

Upon insertion of the left-hand side of Eq. (4.5) for $\sigma$ in the right-hand side of Eq. (4.4), this equation can then be expressed as a complete derivative by writing

$$
\partial_{\xi}\left[(v+E) \sigma+v \int_{c}^{|E|} d x \frac{f(|x|)}{x}\right]=0 .
$$

For physical fronts with $v>0$ and $\sigma \geqslant 0$ [see Eq. (4.3)], we see from Eq. (4.5), that $E$ is a monotonic function of $\xi$,

$$
\operatorname{sgn}\left(\partial_{\xi} E(\xi)\right)=\operatorname{sgn} q(\xi)=\operatorname{sgn} E(\xi)=\operatorname{sgn} E^{+} \text {for all } \xi \text {. }
$$

This allows us to use $E$ as a coordinate instead of $\xi$. According to Eq. (3.7), before the front at $\xi \rightarrow \infty$ the electron density vanishes, so $\sigma^{+}=\sigma\left[E^{+}\right]=0$. Equations (4.6) and (4.7) together then determine $\sigma$ as a function of $E$ as

$$
\sigma[E]=\frac{v}{v+E} \rho_{E^{+}}[E]
$$

with the function

$$
\rho_{E^{+}}[E]=\int_{|E|}^{\left|E^{+}\right|} d x \frac{f(|x|)}{x}=\rho_{\left|E^{+}\right|}[|E|](\geqslant 0) .
$$

The function $\rho_{E^{+}}[E]$ is nothing but the ion density, as can be deduced by inserting $q=\partial_{\xi} E$ into Eq. (4.5) and equating the charge density $q$ with $\rho-\sigma$. The ion density $\rho$ for $D=0$ turns out to be a function of $E$ and $E^{+}$only, and to be independent of the particular front shape parametrized by $v$.

The fields $\sigma, \rho$, and $E$ as a function of $\xi$ can be found by solving the implicit equation for $E=E(\xi)$,

$$
\partial_{\xi} \ln |E|=\frac{\rho_{\left|E^{+}\right|}[|E|]}{v+E},
$$

which can be derived from Eqs. (4.5) and (4.9).

Equation (4.8) immediately shows that physically allowable solutions with $\sigma \geqslant 0$ and $v>0$ must have $v+E \geqslant 0$ for all field values. Because of the monotonicity of $E$ as a function of $\xi$, this is automatically satisfied for PSF with $E^{+}>0$, but for NSF this implies in particular that $v+E^{+} \geqslant 0$; together with $v>0$ we thus have for physical fronts

$$
v \geqslant \max \left[0,-E^{+}\right]
$$

In physical terms, the condition $v \geqslant-E^{+}$expresses that the velocity of uniformly translating fronts must be at least the drift velocity $-E^{+}$of free electrons in the leading edge of the front, where the field is strongest. [Remember that Eq. (4.7) implies that the field is monotonic in space.]

For all values of $v$ obeying the inequality (4.11), the solutions of Eqs. (4.8) and (4.10) are proper, physically allowable solutions for fixed $E^{+}$; within the context of the present model, this illustrates a general feature of front propagation into unstable states, namely that there exists a family of front solutions parametrized by the velocity [42].

In Fig. 2(a), we plot the solution (4.8) for $\sigma$ as a function of $E$ for the fixed value of the velocity $v=2$ in the case that the impact ionization function $f(E)$ is given by the Townsend expression $f_{T}(E)$ of Eq. (2.16) as in the numerical simulations $[8,9]$. Note that in this representation, the state behind the front at $\xi=-\infty$ corresponds to a point on the $\sigma$ axis, and that the front solution $\sigma(\xi), E(\xi)$ is represented in this diagram by the flow along one of the trajectories towards either the positive $E$ axis for PSF or the negative $E$ axis for NSF for $\xi \rightarrow \infty$. Note furthermore that $\sigma$ overshoots the value $\sigma^{-}[=\sigma(\xi \rightarrow-\infty)]$ in the case of NSF. This property as well as the monotonicity of $\sigma[E]$ and accordingly of $\sigma(\xi)$ for positive fronts follows immediately from Eq. (4.8). For NSF, it can also be observed in the three-dimensional simulations of Vitello et al. [9], shown in Fig. 1(c).

The smallest $E^{+}$for which a front solution with $v=2$ is shown in Fig. 1(a), is $E^{+}=-1.999$. For this value of $E^{+}$, $\sigma[E]$ continues to increase till $E \approx E^{+}$and then suddenly decays to zero. A short analytical investigation of Eq. (4.8) shows that this behavior develops into a discontinuity of $\sigma[E]$ at the point $E=E^{+}$for $v=-E^{+} . \sigma[E]$ then increases monotonically up to $f\left(E^{+}\right)$for $E \downarrow E^{+}$and then jumps to zero 
(b)

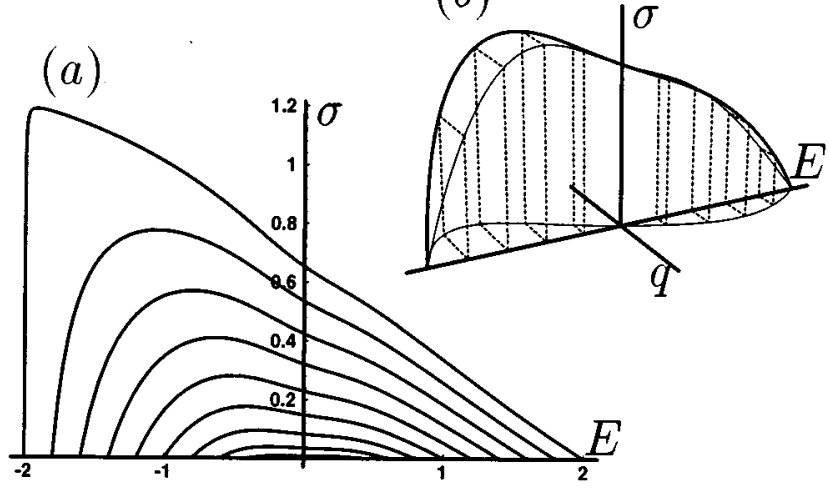

FIG. 2. (a) Uniformly translating fronts for $D=0$ and $v=2$ shown as flows in the two-dimensional $(E, \sigma)$ phase space. Out of each point $\sigma^{-}$on the $\sigma$ axis, there is a PSF flowing to the right and a NSF to the left. Both reach the same value $\left|E^{+}\right|$on the horizontal axis, which also is independent of $v$. Note that NSF have a maximum of $\sigma$ within the front, while PSF have monotonic $\sigma$. Note, also, that no physical fronts (i.e., with $\sigma \geqslant 0$ everywhere) reach a value $E^{+}<-v=-2$, in agreement with Eq. (4.11). ( $b$ ) Sketch of a uniformly translating PSF and NSF for $D \neq 0$ as a flow in threedimensional $(E, \sigma, q)$ phase space. The thick curves indicate the trajectories, while the thin ones show their projection into the $\sigma=0$ and $q=0$ planes. For fixed $v$, there is at each point of the $\sigma$ axis still only one outgoing vector, which can be followed in two antiparallel directions. The $E$ axis is fully attractive for $E>-v$ and then always will be reached. be reached.

discontinuously at $E^{+}$. This shocklike behavior stays unchanged under a parameter change to $\sigma(\xi)$. It is further discussed and motivated in Sec. V.

An immediate consequence of Eqs. (4.8) and (4.9) for the electron and ion density is that the value $\sigma^{-}$behind the front (where $E \rightarrow 0$ ) is a simple function of the value $E^{+}$of the field ahead of the streamer profile:

$$
\sigma^{-}\left(E^{+}\right)=\rho_{\left|E^{+}\right|}[0]=\int_{0}^{\left|E^{+}\right|} d x \frac{f(|x|)}{x} .
$$

The virtue of this expression for the electron density $\sigma^{-}$far behind the front, as well as of the expression (4.9) for the ion density $\rho$ throughout the whole front, is that it is independent of the velocity $v$, hence independent of whichever front profile is selected, provided that the limit $D \rightarrow 0$ is smooth. We shall see later that this $D=0$ result remains relatively accurate for NSF fronts with $D \leqslant 1$, and compare it to the results of the simulations [8,9] in Sec. VI. For PSF, on the other hand, the above result will turn out to be less relevant due to the nonperturbative nature of the limit $D \rightarrow 0$ in this case.

For the Townsend function $f_{T}(|x|)$ [Eq. (2.16)] the function $\sigma^{-}\left(E^{+}\right)$can be expressed as

$$
\begin{aligned}
\sigma^{-}\left(E^{+}\right)_{T} & =\left|E^{+}\right| E_{2}\left(\left|E^{+}\right|^{-1}\right) \\
& =f_{T}\left(\left|E^{+}\right|\right)-E_{1}\left(\left|E^{+}\right|^{-1}\right),
\end{aligned}
$$

where $E_{n}(z)$ is the exponential integral [43].

We finally note that the second form of Eq. (4.13) shows that $\sigma^{-}$approaches $f_{T}$ for large fields, since $f_{T} \gg E_{1}$ for $E^{+} \gg 1$. For $E^{+}$of order unity, $\sigma$ and $f_{T}$ are still of the same order, and this shows (for small $D$ ) that the growth rate (3.3) of long wavelength unstable modes in the nonionized state is comparable to the damping rate (3.5) of stable modes in the plasma behind a NSF. For small fields, the strict bounds on $E_{2}$ [43] show that $\sigma^{-} \approx E^{+} f_{T}\left(E^{+}\right)$, so that the approximate equivalence of these two time scales does not hold for $E^{+} \ll 1$, but in the small field range our starting model is not very realistic anyway, because of the neglect of stabilizing recombination terms.

\section{B. $D \neq \mathbf{0}$ front solutions}

For $D \neq 0$, we cannot obtain the uniformly translating solutions analytically. Moreover, perturbation theory around the $D=0$ case is not simply possible, as $D$ appears in front of the highest derivative in Eq. (4.2), so the diffusion term acts as a singular perturbation. As a consequence, Eqs. (4.2) reduce to a set of two coupled first order ODE's for $D=0$, while three are required for $D \neq 0$. However, we can still easily demonstrate the existence of a one-parameter family of uniformly translating front solutions for $D \neq 0$ through standard counting arguments for ODE's. Building on the results of such an analysis, the solutions can then be constructed by integrating numerically in a stable direction, using so-called "shooting methods" [44].

To perform the analysis, it is convenient to write the equations as a set of three coupled first order ODE's. There is some freedom for the choice of the third variable: The standard choice would be $\sigma^{\prime}=\partial_{\xi} \sigma$, but for the discussion of the singular limit as well as for numerical stability, the charge density $q$ has turned out to be the most convenient choice. The ODE's (4.2) then become

$$
\begin{aligned}
& \partial_{\xi} \sigma=-\frac{\sigma E-v q}{D}, \\
& \partial_{\xi} E=q, \\
& \partial_{\xi} q=-\frac{\sigma f(|E|)}{v}+\frac{\sigma E-v q}{D} .
\end{aligned}
$$

Just as we thought of the profiles for $D=0$ as describing flow in a two-dimensional $(\sigma, E)$ phase space, we can now think of Eqs. (4.14) as describing a flow in a threedimensional $(\sigma, E, q)$ phase space. The velocity $v$ just plays the role of a parameter in the flow equations, while $\xi$ again plays the role of a timelike variable — see the sketch in Fig. 2(b).

The steady states of the full PDE's discussed in Sec. III correspond to fixed points of the flow: the points $(\sigma, 0,0)$ on the $\sigma$-axis are fixed points of the flow (4.14), that correspond to homogeneously ionized plasma states, while the $E$ axis is a line of fixed points $(0, E, 0)$ each of which corresponds to a nonionized state with $\sigma=\sigma^{\prime}=0$ and $E \neq 0$.

A uniformly translating front solution now corresponds to the existence of a trajectory in this phase space that starts at "time" $\xi=-\infty$ on the $\sigma$ axis and flows to the $E$ axis for $\xi \rightarrow \infty$. The multiplicity of such solutions (i.e., whether they exist as discrete sets, or, e.g., as a one- or two-parameter family) can be determined as follows. If we linearize the 
flow near an arbitrary point $\left(\sigma^{-}, 0,0\right)$ on the $\sigma$ axis by writing $(\sigma, 0,0)=\left(\sigma^{-}, 0,0\right)+A \exp \left(-\Lambda^{-} \xi\right)$, we find the eigenvalue equation

$$
\Lambda^{-}\left(\Lambda^{-2}-\Lambda^{-} \frac{v}{D}-\frac{\sigma^{-}}{D}\right)=0 .
$$

The fact that there is a zero eigenmode is a consequence of the fact that the $\sigma$ axis is a line of fixed points. For the two nontrivial eigenvalues [which correspond to the linearized modes (3.5) about the ionized and screened region by equating $i \mathbf{k} \cdot \hat{\mathbf{x}}=-\Lambda^{-}$and $\left.\omega^{-}=\Lambda^{-} v\right]$ we have

$$
\Lambda_{ \pm}^{-}=\frac{v \pm \sqrt{v^{2}+4 D \sigma^{-}}}{2 D} \text {. }
$$

The eigenvalue $\Lambda_{+}^{-}$is positive, and hence gives a decaying exponential; thus points along the corresponding eigendirection flow into the $\sigma$ axis as $\xi$ increases. The eigenvalue $\Lambda_{-}^{-}$, on the other hand, is negative and hence corresponds to an unstable eigendirection, with flow away from the axis. This implies that at each point $\left(\sigma^{-}, 0,0\right)$ on the $\sigma$ axis, there is, for fixed $v$, a unique eigendirection $\left(-\Lambda_{-}^{-}, 1, \Lambda_{-}^{-}\right) E_{1}$ along which the flow is away from the axis. This flow can be followed in two antiparallel directions, determined by the sign of $E_{1}$. The one flowing towards positive values of $E$ is the beginning of a PSF front profile, the one flowing towards negative $E$ is the beginning of an NSF profile. From these eigendirections, one derives that for PSF with field perturbations $E_{1}>0$, the electron density decreases close to the $\sigma$ axis, while for NSF it increases. Accordingly, before reaching $\sigma=0$ for $\xi \rightarrow \infty$, a NSF profile has at least one maximum of $\sigma$, while a negative one can be (and is) monotonic. This generalizes our result for $D=0$, and is consistent with the findings of Vitello et al. [9] shown in Fig. 1(c). The physical origin of the maximum of $\sigma$ in the rear end of the NSF profile is the screening of the field: Due to the low ionization rate in an already fairly suppressed field the ion density has already almost acquired its final value, so the electron density has to overshoot its asymptotic value $\sigma^{-}$so as to make $\partial_{\xi} E<0$. The screening behind a PSF happens by suppressing the electron density faster than the ion density for increasing $\xi$, and so there $\sigma$ is monotonic.

Let us now investigate the stability of the flow near a point $\left(0, E^{+}, 0\right)$ on the $E$ axis. Upon linearizing the flow equations (4.14) and writing the $\xi$ dependence of the perturbations in the form $\exp \left(-\Lambda^{+} \xi\right)$, we find the eigenvalue equation

$$
\Lambda^{+}\left(\Lambda^{+2}-\Lambda^{+} \frac{v+E^{+}}{D}+\frac{f\left(\left|E^{+}\right|\right)}{D}\right)=0
$$

Again, there is a zero eigenvalue due to the fact that the whole $E$ axis is a line of fixed points. The two nontrivial eigenvalues are

$$
\Lambda_{ \pm}^{+}=\frac{v+E^{+} \pm \sqrt{\left(v+E^{+}\right)^{2}-4 D f\left(\left|E^{+}\right|\right)}}{2 D} .
$$

These eigenvalues can be related to Eq. (3.3) in the same way as Eq. (4.15) could be related to (3.5). For $v+E^{+}>0$, the real parts of these eigenvalues are always positive, so that both eigendirections are stable. In other words, for $E^{+}>-v$, all points near the $E$ axis flow towards this axis in slightly more technical terms, there is a two-dimensional stable manifold flowing into each of these points on the $E$ axis. For $E^{+}<-v$, the flow is away from the $E$ axis, and fronts with $v+E^{+}<0$ cannot be constructed. This generalizes Eq. (4.11) to $D \neq 0$.

The existence of a one-parameter family of fronts with velocity $v>-E^{+}$can now simply be understood as follows. As we saw before, there is one unique PSF and one unique NSF trajectory flowing out of each point on the $\sigma$ axis for fixed $v$ and $D$. Since the flow defined by Eqs (4.14) is continuous, we can expect each trajectory to extend smoothly [45]. Once the flow gets near the $E$ axis, we know from the above analysis that the trajectory will be attracted completely to the axis, provided $v$ is large enough. Thus, for each $\sigma^{-}$ and $v$, there will exist two unique trajectories, i.e., a unique PSF solution and a unique NSF solution. Since each of these trajectories flows into a unique point on the $E$ axis, the flow equations implicitly define a unique relation of the form $\sigma^{-}=\sigma^{-}\left(v, E^{+}\right)$for each of the two types of fronts. For a given value of $E^{+}$, we thus have a one-parameter family of front solutions, parametrized by $v$.

There are two important properties of the front solutions associated with their asymptotic large $\xi$ behavior. First of all, we note that according to Eq. (4.18) the eigenvalues $\Lambda_{ \pm}^{+}$are only real for

$$
v \geqslant v^{*} \equiv-E^{+}+2 \sqrt{D f\left(\left|E^{+}\right|\right)} .
$$

This implies that the corresponding front profiles can certainly not approach the asymptotic state $\sigma=0$ ahead of the front in a monotonic way for $v<v^{*}$ : When the eigenvalues are complex, the front profiles have an oscillatory tail of the form $\exp \left[-\left(\operatorname{Re} \Lambda^{+}\right) \xi\right] \cos \left[\left(\operatorname{Im} \Lambda^{+}\right) \xi\right]$. Clearly, this violates the physical condition that the electron density $\sigma$ should remain positive, so solutions with $-E^{+}<v<v^{*}$ are physically excluded: $v^{*}$ denotes, in the present case, the smallest velocity of physically allowable uniformly translating front solutions.

The identification of $v^{*}$ as a bound on the velocity of physically allowed front profiles depends only on the structure of the eigenvalues $\Lambda^{+}$associated with the linear flow near unstable states. There is a second, nonlinear, way in which the range of physically allowed values of $v$ can be bounded. To understand this, note that for any $v \geqslant v^{*}$, the asymptotic decay of $\sigma(\xi)$ for $\xi \rightarrow \infty$ for a uniformly translating profile will be

$$
\sigma(\xi)=A_{-} e^{-\Lambda_{-}^{+} \xi}+A_{+} e^{-\Lambda_{+}^{+} \xi}+\text { h.o.t. }
$$

with real coefficients $A_{-}$and $A_{+}$. Here, h.o.t. stands for higher powers of the two exponentials generated when expanding the equations to higher than linear order in the variables. Clearly, the smallest eigenvalue $\Lambda_{-}^{+}$governs the asymptotic decay of the profile provided $A_{-} \neq 0$. That $A_{-}$will generically be nonzero for an arbitrary velocity $v$ follows again from the counting argument above for the flow in phase space: Each PSF and NSF trajectory flowing out of a point on the $\sigma$ axis is unique, and hence there is no freedom 
to impose an additional condition $A_{-}=0$ close to the $E$ axis. Furthermore, the coefficients $A_{-}$and $A_{+}$depend on the full global nonlinear behavior of the flow, and hence they depend implicitly on $v$.

There might exist, however, particular velocities $v^{\text {part }}>v^{*}$, for which

$$
A_{-}\left(v^{\text {part }}\right)=0 .
$$

For discussing these we invoke again a continuity argument for the front properties as a function of $v$. We expect a very slowly decaying, nearly homogeneous uniformly translating front solution to have a non-negative density everywhere, and to have a very large velocity, since the velocity of a profile is essentially inversely proportional to its slope in the limit of small slopes. [So indeed the roots $\Lambda_{-}^{-}$given by Eq. (4.16) and $\Lambda_{-}^{+}$given by Eq. (4.18) vanish in the limit that $v$ becomes large.] So for large $v$ we expect to find physical solutions. These are characterized by $A_{-}>0$ in the leading edge of the front. Decreasing $v$ continuously, we either reach $v=v^{*}$ smoothly with still $A_{-}>0$, or we reach the first particular velocity, $v_{1}^{\text {part }}$, where $A_{-}$vanishes. In the latter case, we expect by continuity $A_{-}(v)<0$ for $v<v_{1}^{\text {part }}$. This implies that then $\sigma$ approaches zero from below, i.e., that the front solution is unphysical. Below the next $v_{2}^{\text {part }}$, we expect the electron density to develop two zeros and so forth. The largest $v^{\text {part }}$, if it exists, thus plays the role of the nonlinear front velocity $v^{\dagger}[24]$,

$$
v^{\dagger}=\max \left\{v^{\text {part }} \mid A_{-}\left(v^{\text {part }}\right)=0\right\}
$$

for a given $E^{+}$. [Note that if $\Lambda_{-}^{+}<0.5 \Lambda_{+}^{+}$the higher order terms in Eq. (4.20) of order $\exp \left(-2 \Lambda_{-}^{+} \xi\right)$ are actually larger than the second term $\exp \left(-\Lambda_{+}^{+} \xi\right)$. This does not change our argument, though, as the prefactor of this second order term will vanish if $A_{-}$vanishes.]

At the velocity $v=v^{\dagger}$ or at any $v=v^{\text {part }}$, the flow in phase space approaches the $E$ axis along the eigenvector where the flow is most rapidly contracting. The trajectory corresponding to the nonlinear front solution is therefore more appropriately referred to as a strongly heteroclinic orbit, where heteroclinic indicates that it is a trajectory from one fixed point to another one. The existence and properties of strongly heteroclinic orbits have recently been under active investigation [46].

Such a velocity $v^{\dagger}$, if it exists, bounds the continuum of velocities of physical uniformly translating solutions from below, and thus replaces the earlier bound $v^{*}$ derived from linearizing the equations in the leading edge of the front.

\section{Nonlinear front solutions for PSF}

For NSF, the bounding velocity $v^{*}$ given by Eq. (4.19) is always positive. Moreover, by integrating the flow equations (4.14) numerically and searching for particular solutions for which, according to Eq. (4.21), $A_{-}\left(v^{\text {part }}\right)=0$, we have convinced ourselves that there are no such solutions for any $D$ $\neq 0$ and $E^{+}<0$. Hence, the smallest velocity of physical NSF solutions is always $v^{*}$, for any value of the parameters.

For PSF, on the other hand, the situation is very different, since $v^{*}<0$ for $\left(E^{+}\right)^{2}>4 D f\left(\left|E^{+}\right|\right)-$for the Townsend

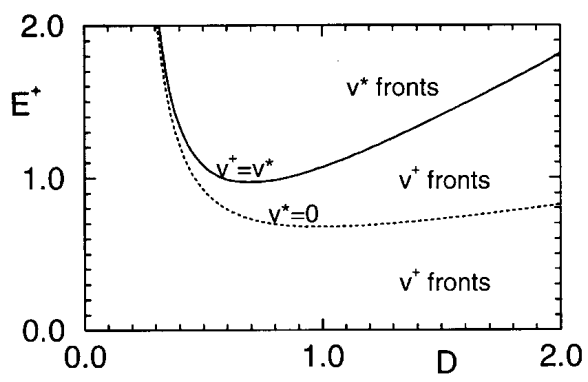

FIG. 3. Phase diagram for PSF as a function of $D$ and $E^{+}$. Above the solid line the lowest speed of physical front solutions is given by $v^{*}$, below the line by $v^{\dagger}$ corresponding to the smallest speed of physical front solutions. Accordingly, the selected front speed is $v^{*}$ above the solid line (linear marginal stability regime), and $v^{\dagger}$ below the solid line (nonlinear marginal stability regime). The dotted curve indicates $v^{*}=0$ and is a lower bound for the crossover to $v^{\dagger}$ behavior of the selected fronts.

function (2.16), this happens for $D \leqslant 0.25 E^{+} e^{1 / E^{+}}$, hence for any $E^{+}$for $D \leqslant 0.68$. In particular for PSF at small $D$ the question therefore arises whether there are nonlinear front solutions defined by Eq. (4.21) and (4.22) with $v^{\dagger}>0$. The results of a numerical search for such solutions are shown in Fig. 3, as a function of $D$ and $E^{+}$. Below the full line in this diagram, there exists indeed a nonlinear front $v^{\dagger}>v^{*}$, whereas above this line $v^{*}$ denotes the smallest velocity of physical front solutions. While these results have been obtained numerically, the existence of a single (unique) particular solution with $A_{-}\left(v^{\text {part }}\right)=0$ in the limit $D \rightarrow 0$ can be demonstrated analytically. Since a full discussion of these results will be given elsewhere [47], we confine ourselves here to a brief outline of the arguments that also demonstrate the singular nature of these solutions for $D \rightarrow 0$.

If we take the limit $D \rightarrow 0$ with $v$ fixed, assuming no nontrivial scaling of the variables $\sigma, E$, and $q$ and of the spatial coordinate $\xi$, Eqs. (4.14) can easily be shown to reduce to those studied in Sec. IV A for $D=0$. Hence, we can recover in this way the family of front solutions obtained there. Any particular solution, on the other hand, for which $A_{-}\left(v^{\text {part }}\right)=0$, decays according to Eq. (4.20) as $\exp \left(-\Lambda_{+}^{+} \xi\right)$ as $\xi \rightarrow \infty$. Since $\Lambda_{+}^{+} \propto D^{-1}$ for $D \rightarrow 0$, such a particular front solution becomes extremely steep as $D \rightarrow 0$ : its gradients diverge as $1 / D$ so that the diffusion term can still overcome the drift term as $D \rightarrow 0$. That the velocity of such a solution must also have a nontrivial scaling in this limit can be seen from the third equation of Eq. (4.14), written in the form

$$
\partial_{\xi} q=\sigma\left(-\frac{f(|E|)}{v}+\frac{E}{D}\right)-\frac{v}{D} q
$$

Any nontrivial scaling of this equation in the limit $D \rightarrow 0$ can only occur if the first term between large parentheses remains of the same order as the other two, which diverge as $1 / D$. This is only possible if $v$ scales as $D$. In this limit, the third term can then be neglected, and since $\partial_{\xi} q$ has to change sign in the front region (as the charge density $q$ vanishes as $\xi \rightarrow \pm \infty)$, there must be an intermediate value $\hat{E}<E^{+}$of the field for which $v=D f(|\hat{E}|) / \hat{E}$. 
Now that we know the scaling of the spatial gradient of the velocity of such particular front profiles for $D \rightarrow 0$, one easily convinces oneself that the electron and charge density of these solutions must diverge as $1 / D$ in this limit. To study the existence of such possible solutions, it is therefore convenient to introduce new variables and coordinates according to

$$
x=D \tilde{x}, \quad v=D \widetilde{v}, \quad \xi=D \widetilde{\xi}, \quad \sigma=\widetilde{\sigma} / D, \quad q=\widetilde{q} / D,
$$

with $E$ and $\tau$ unchanged. In these new variables, the flow equations (4.14) become

$$
\begin{aligned}
& \partial \widetilde{\xi} \widetilde{\sigma}=-\widetilde{\sigma} E+D \widetilde{v} \widetilde{q}, \\
& \partial \widetilde{\xi} E=\widetilde{q}, \\
& \partial \widetilde{\xi} \widetilde{q}=\widetilde{\sigma}\left(E-\frac{f(|E|)}{\widetilde{v}}\right)-D \widetilde{v} \widetilde{q} .
\end{aligned}
$$

The limit $D \rightarrow 0$ can now be taken simply by leaving out the term $D \tilde{v} \widetilde{q}$ in the first and last equation. We will show elsewhere [47] that the resulting equations have one unique physical front solution thus fixing one particular value of the scaled velocity $\widetilde{v_{1}}$, and in view of the scaling (4.24) and the scaling of the eigenvalues $\Lambda_{ \pm}^{+}$, this solution must have $A_{-}\left(\widetilde{v_{1}}\right)=0$. This solution is therefore precisely the $D \rightarrow 0$ limit of the nonlinear front solution with velocity $v^{\dagger}=\widetilde{v_{1}} D$. Furthermore, since the limit $D \rightarrow 0$ is smooth for Eqs. (4.25), this shows that there exists a nonlinear front solution with $v^{\dagger}>0$ for any $E^{+}$and nonzero but small $D$. Due to the singular scaling (4.24), the corresponding front solutions are determined by ODE's that have a different structure from those studied for $D=0$ in Sec. IV A, and therefore these nonlinear front solutions cannot be obtained perturbatively from the latter class of solutions - of course, the latter class of solutions still exists for $D \neq 0$, in agreement with the counting arguments given earlier, but these now correspond to a singular limit of Eqs. (4.25). The significance of these nonlinear front solutions lies in the fact that they will turn out to be the selected fronts that dominate the dynamics of PSF in the physically important range $0.1 \leq D \leq 0.3$.

The nonlinear front solution can be constructed numerically very easily by integrating Eqs. (4.25) using standard numerical "double shooting" routines [44]. Figure 4 shows our numerical results for the smallest physical velocity, $\max \left(v^{\dagger}, v^{*}\right)$ in the case that the ionization function is given by the Townsend expression. The scaled velocities $v^{\dagger} / D$ and $v^{*} / D$ are plotted; in agreement with our arguments above, the scaled velocity $v^{\dagger} / D$ of the nonlinear front solution approaches a finite limit as $D \rightarrow 0$. Furthermore, the ratio $v^{\dagger} / D$ hardly varies with $D$ in the physical range $0.1 \leq D \leq 0.3$, and for small fields $E^{+}$, the scaled velocity $v^{\dagger} / D$ becomes exponentially small, in agreement with the bound $v^{\dagger} / D<E^{+} \exp \left(-1 / E^{+}\right)$that follows from the observations discussed after Eq. (4.23) above.

We finally note that our numerical routines have not only allowed us to obtain the results show in Figs. 2 and 4, but have also enabled us to verify numerically all the statements made above about the multiplicity of solutions, the parameter

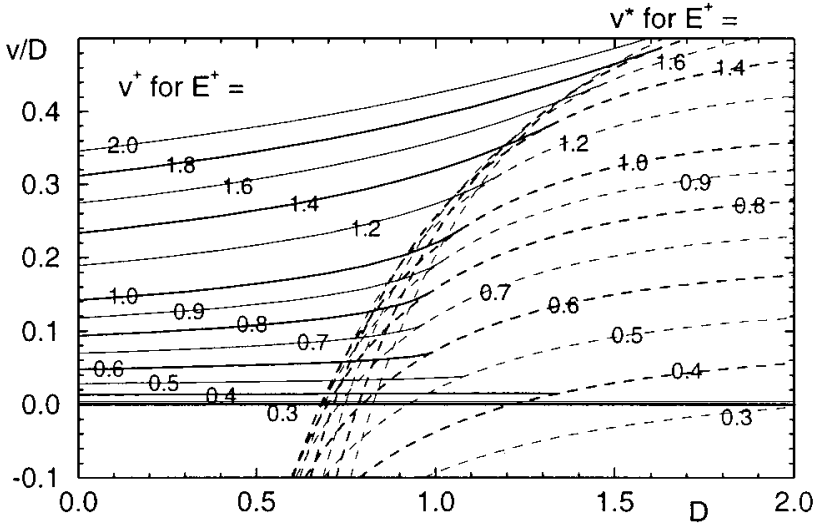

FIG. 4. $\tilde{v}^{\dagger}=v^{\dagger} / D$ (solid) and $\tilde{v} *=v^{*} / D$ (dashed lines) as a function of $D$ for $E^{+}=0.3-1.0$ in steps of 0.1 , and for $E^{+}=1.0$ - 2.0 in steps of $0.2 . \widetilde{v}^{\dagger}$ depends only weakly on $D$, i.e., the physical front velocity $v^{\dagger}$ is approximately proportional to $D$. At $\widetilde{v}^{\dagger}\left(E^{+}, D_{c r}\left(E^{+}\right)\right)=\widetilde{v} *\left(E^{+}, D_{c r}\left(E^{+}\right)\right)$, the selected front crosses over from $v^{\dagger}$ to $v^{*}$; the $v^{\dagger}$ solutions disappear. Plotting $D_{c r}\left(E^{+}\right)$in the $\left(E^{+}, D\right)$ plane yields the solid curve in the phase diagram of Fig. 3, while the zeros of $v^{*}$ determine the dotted curve in Fig. 3.

ranges for physical fronts, the monotony properties, the singular behavior of the small $D$ PSF limit, and the persistence of the family of front solutions for $D \rightarrow 0$.

\section{SELECTION OF THE ASYMPTOTIC FRONT}

\section{A. Front propagation into unstable states}

We have seen that the nonionized state into which the streamer fronts propagate is an unstable state, that the homogeneous weakly ionized plasma is a stable state, and that there is a family of uniformly translating front solutions connecting the two. The existence of a family of front solutions is a generic feature of front propagation into unstable states. $\mathrm{We}$, therefore, briefly recall what is known in the literature for analogous problems and then translate this experience to the streamer problem. The prototype equation for studies of this type of front propagation is

$$
\partial_{t} u=\partial_{x}^{2} u+g(u),
$$

where $g(u)$ is some nonlinear function which satisfies

$$
g(0)=0, \quad g(1)=0, \quad g^{\prime}(0)>0, \quad g^{\prime}(1)<0 .
$$

Note that these relations imply that the "state" $u=0$ is unstable, and that the "state" $u=1$ is stable. The study of the propagation of fronts into the unstable state $u=0$ in this equation dates back to the early work of Kolmogorov et al. [33] and Fisher [34] in the context of population dynamics. Later Gel'fand [48] studied a particular example of a function $g(u)$ motivated by combustion. The mathematical research on this equation culminated in the work by Aronson and Weinberger [31], who rigorously solved the front propagation problem for Eq. (5.1). In particular, they proved that any initial perturbation that is nonzero only in a finite part of space approaches a unique uniformly translating front solution with velocity $v_{f}$ in the long time limit. If $g^{\prime \prime}(u)<0$ for all $u, v_{f}$ equals $v^{*}=2 \sqrt{g^{\prime}(0)}$ (derived from linearizing in 
the tip of the front), while for general $g(u), v_{f}$ approaches either $v^{*}$ or some $v^{\dagger}>v^{*}$. We refer to the literature for a detailed discussion of this work $[31,32]$.

The velocities $v^{*}$ and $v^{\dagger}$ of the above problem directly correspond to our $v^{*}$ (4.19) and $v^{\dagger}$ (4.22), since they are also the smallest velocities, which still allow for uniformly translating fronts with $u \geqslant 0$ everywhere. So if $u$ is interpreted as a population density or a chemical concentration, the selected front for every sufficiently localized initial state is the slowest physical uniformly translating front. In other interpretations no physical constraints bind $u$ to positive values. Nevertheless the selected velocity stays the same. In this case, one can prove that every front with smaller velocity is dynamically unstable [21], i.e., that the selected front is marginally stable. The slowest physical or stable solution, which is selected, coincides with the steepest physical or stable one.

In the last decade, it has been recognized that several aspects of the front selection problem encountered for the nonlinear diffusion equation (5.1) seem to have more general validity. Certain scenarios, justified by heuristic arguments but lacking a detailed mathematical proof, were formulated and numerically tested on more complicated PDE's that were often of higher order in the spatial derivatives [49,21-25]. Some of the equations studied lead to nonuniformly translating fronts that leave a nontrivial spatially periodic state behind $[49,21,23,24,50]$. A particular scenario is the one distinguishing between the so-called linear marginal stability regime where $v_{f}=v^{*}$ and the nonlinear marginal stability regime where $v_{f}=v^{\dagger}[21-24]$. These names stem from the fact that in this formulation, the two regimes of front selection are related to the stability properties of the front solutions - in both cases, the selected front separates stable front solutions from unstable ones. Applied to Eq. (5.1), this scenario just provides an intuitive explanation of all the wellknown mathematical results. For plasma physicists, it is worth mentioning that dynamics in the linear marginal stability regime is related to that determined by the "pinch point analysis" which was developed in plasma physics in the late 1950s [51,52,23].

\section{B. Predictions for streamer fronts}

By extending the arguments in the appendix of [23], one may show that in the streamer case just like in the case of the above problem (5.1), all physical solutions, i.e., all solutions with $\sigma \geqslant 0$ resp. $u \geqslant 0$ everywhere, are stable. For a detailed discussion, we refer to [47]. It can be argued [22,23], and proven for Eq. (5.1) [21], that a sufficiently localized initial condition will approach the physical uniformly translating front, which is closest in "phase space," i.e., the steepest one. Both for Eq. (5.1) and for the streamer equations, the steepest uniformly translating physical front is uniquely defined. It is also the slowest one.

We can immediately prove this when initially $\sigma(x, \tau=0)=0$ for $x>x_{c}$ for streamer fronts with $D=0$ : In general, there is a front solution for every $v \geqslant \max \left[0,-E^{+}\right]$, but now the only way in which the electrons can enter the range $x>x_{c}$ is through electron drift with velocity $-E^{+}$. Clearly, therefore, the asymptotic front speed of a NSF can only be $-E^{+}$, while a PSF cannot propagate at all. If the initial electron density, however, decays exponen- tially, the local electron density grows by drift and ionization, and the front can move quicker than $-E^{+}>0$ for a NSF.

For $D \neq 0$, we will here only conjecture the analogous statements as follows, and we will test them numerically in Sec. VI.

(i) Selected front velocity. If the initial conditions are sufficiently localized, the selected front is the slowest physically acceptable front solution, i.e., the slowest front profile for which $\sigma(\xi) \geqslant 0$ for all $\xi$. In view of the discussion of Sec. $\mathrm{IV}$, this means that the selected front velocity $v_{f}$ is predicted to be

$$
v_{f}=v^{*}=-E^{+}+2 \sqrt{D f\left(\left|E^{+}\right|\right)},
$$

except when there exists a nonlinear front solution satisfying Eq. (4.22). In that case,

$$
v_{f}=v^{\dagger}
$$

Note that the result (5.3) $\left(v^{*}\right.$ is the linear marginal stability value in the terminology of $[22,23]$ ) is an explicit expression for $v_{f}$ in terms of parameters associated with the linear instability of the unstable state only. On the other hand, the existence of a nonlinear front and the value of $v^{\dagger}$ (the nonlinear marginal stability value) depends on the whole nonlinear behavior of the flow equations (4.14).

(ii) Localized initial conditions. Initial conditions are sufficiently localized if their spatial decay is faster than the asymptotic decay associated with the smallest eigenvalue of the selected profile, i.e., if

$$
\sigma(x, \tau=0)<C e^{-\Lambda_{-}^{+}\left(v^{*}\right) x}
$$

or

$$
\sigma(x, \tau=0)<C e^{-\Lambda_{-}^{+}\left(v^{\dagger}\right) x}, \text { for } x \rightarrow \infty,
$$

depending on whether the selected front is $v^{*}$ or $v^{\dagger}$. Here $C$ is an arbitrary constant, and $\Lambda_{-}^{+}\left(v^{*}\right)\left[=\Lambda_{+}^{+}\left(v^{*}\right)\right]$ and $\Lambda_{-}^{+}\left(v^{\dagger}\right)$ are given by Eq. (4.18).

(iii) Nonlocalized initial conditions. If an initial condition does not obey conditions (5.5) or (5.6), faster front speeds are possible. In particular, if initially $\sigma(x, \tau=0)$ $\sim \exp (-\Lambda x)$, with $\Lambda<\Lambda_{-}^{+}\left(v^{*}\right)$ or $\Lambda<\Lambda_{-}^{+}\left(v^{\dagger}\right)$, whichever regime applies, then the front speed is given by

$$
v=-E^{+}+D \Lambda+\frac{f\left(\left|E^{+}\right|\right)}{\Lambda},
$$

which is obtained by solving Eq. (4.17) for $v$ in terms of $\Lambda$.

We now combine the analytic and numeric findings from Sec. IV with the selection rules above to quantitative predictions for asymptotic fronts, which evolve from sufficiently localized initial conditions, in the case that the impact ionization is given by the Townsend expression (2.16).

$N S F$. For NSF, we numerically have not found any nonlinear fronts for any $D$ and $E^{+}$, so our simple yet powerful prediction is that for $\mathrm{NSF} v_{f}=v^{*}$ with $v^{*}$ given by Eq. (5.3). In principle it is possible that for ionization functions $f(E)$ other than the Townsend function (2.16), there can be nonlinear front solutions also in the NSF regime. In practice, 

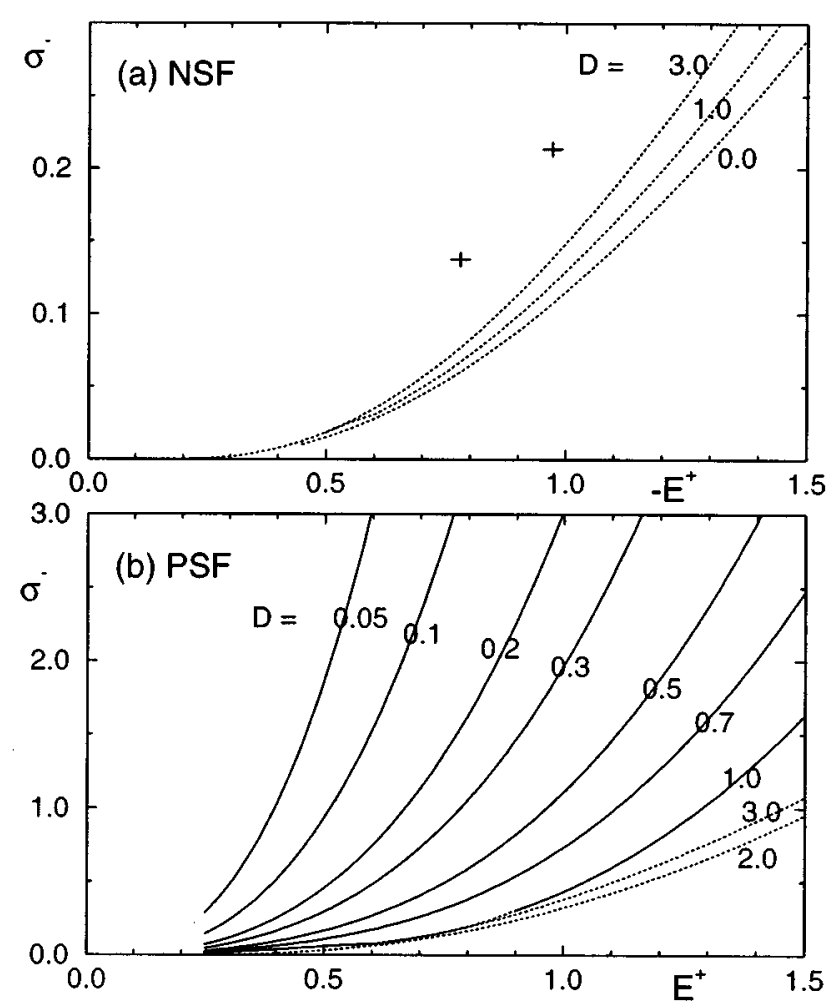

FIG. 5. Electron density $\sigma^{-}$behind the planar selected front as a function of the field $E^{+}$before the front for several $D$; dotted: $v^{*}$ fronts; solid: $v^{\dagger}$ fronts. (a) NSF: For $v^{*}$ fronts, $\sigma^{-}$depends only weakly on $D$. Results for $D=0,1,3$ are shown. Crosses: Extrapolation of $\sigma^{-}\left(E^{+}\right)$for $D=0.1$ for the curved fronts of the 3D simulations [9]. (b) PSF results for $D=0.05,0.1,0.2,0.3,0.5,0.7,1,2$, 3. For $v^{\dagger}$ fronts, $\widetilde{\sigma}^{-}=$const $+O(D)$, i.e., $\sigma^{-}\left(E^{+}\right)$is approximately proportional to $1 / D$.

we expect, however, that this will not be the case for physically reasonable functions $f(E)$, i.e., for functions consistent with Eq. (2.17).

Once the predicted velocities are known, the value $\sigma^{-}$of the electron density behind the streamer head is obtained from the numerical integration of the flow equations. The results of these calculations are shown in Fig. 5(a). Since for NSF, the limit $D \rightarrow 0$ is smooth, also $\sigma^{-}$depends only weakly on $D$ for $D \leq 1$, so that the $D=0$ prediction (4.13) is quite accurate for realistic values of the diffusion coefficient.

At the predicted values of the selected front velocity, the width of the front region can be obtained directly from our numerical solutions of the flow equations. We have somewhat arbitrarily defined the width $w$ as the distance between the points where $\sigma$ is $90 \%$ and $10 \%$ of the value $\sigma^{-}$. As Fig. 6 shows for NSF fronts with $D=0.1$, this front width is typically of order 3 for field values of order unity. This confirms again that in the small $D$ limit the impact ionization length $\alpha_{0}^{-1}$ sets the inner scale of streamer fronts. Furthermore, we find that our numerical data are well fitted by the expression $w \approx 6 / \Lambda_{ \pm}^{+}\left(v^{*}\right)$, which shows that the front width simply scales with the spatial decay rate $\Lambda_{+}^{+}\left(v^{*}\right)$ $=\Lambda_{-}^{+}\left(v^{*}\right)$ of the streamer profile in the leading edge. NSF fronts always have a maximum of the electron density within the front.

$P S F$. As we saw in Sec. IV, for PSF with $D \leq 0.9$, there

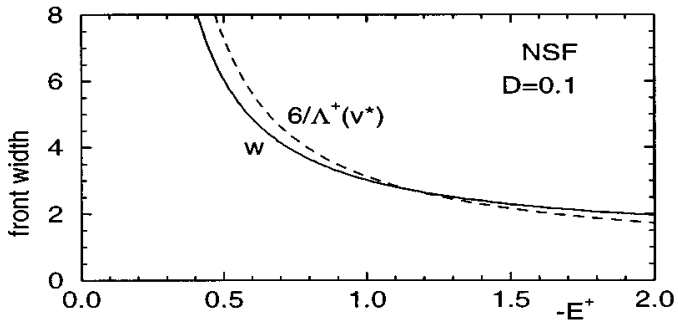

FIG. 6. Width $w$ of the front profile (measured between points with $0.1 \sigma^{-}$and $0.9 \sigma^{-}$) as a function of $E^{+}$for the selected NSF fronts with $D=0.1$. The dashed line is given by $w=6 / \Lambda_{-}^{+}\left(v^{*}\right)$.

always is a nonlinear front solution with velocity $v^{\dagger}>v^{*}$. The prediction is that in this range the selected front solution is the nonlinear front solution, i.e., $v_{f}=v^{\dagger}$. Values of $v^{\dagger}$ as a function of $D$ and for several values of $E^{+}$were already given in Fig. 4. We also saw before that these nonlinear front solutions are singular in the limit $D \ll 1$, where $v^{\dagger} \approx D \tilde{v}^{\dagger}(D=0)$ and $\sigma^{-} \approx \widetilde{\sigma}^{-}(D=0) / D$. The resulting predictions for $\sigma^{-}$are shown in Fig. 5(b).

The fact that the dimensionless inner decay length of these nonlinear fronts scales as $D$ implies that the physical decay length of such solutions is $D / \alpha_{0}=D_{e} /\left(\mu_{e} E_{0}\right)$, i.e., is given by the electron diffusion length. However, since simultaneously the electron density $\sigma^{-}$diverges as $1 / D$, the total front width $w$ defined above still approaches a finite limit as $D \rightarrow 0$ in units of the ionization length $\alpha_{0}^{-1}$.

We finally note that the front propagation problem posed by the one-dimensional streamer equations has a number of interesting differences and similarities with the Aronson Weinberger front propagation problem (5.1). In particular, it can be hoped that techniques of strict bounds developed for the time development of these fronts [31] as well as for the nonlinear front velocity $v^{\dagger}$ [46] might be also applicable to planar streamer fronts.

\section{NUMERICAL TESTS OF THE PREDICTIONS}

We have tested the predictions listed in Sec. V by numerically integrating the PDE's (3.10) and (3.11) forward in time. Our computer program is a finite difference code with a time integration which is based on a semi-implicit method.

We have performed an extensive search through parameter space, varying $D$ between 0.02 and 3 , and $\left|E^{+}\right|$between 0.3 and 10. All our numerical studies of the dynamics fully confirm our predictions for fronts, and therefore we only present a sample of our results that illustrate the important features.

All the simulations of the initial value problem, which we present in the remaining figures, have initially a field $E=-1$ constant in space. We keep the field constant in time in the nonionized region. The simulations of Figs. 7-10 start with the same localized initial ionization seed, a Gaussian profile for the electron density,

$$
\sigma(x, t=0)=0.01 \exp -\left(x-x_{0}\right)^{2} .
$$

Figure 7 shows a run for $D=1$ and times $t=0-130$ in time steps $\Delta t=2$. As can be seen, the small ionization seed near $x_{0}=50$ initially grows while drifting to the right in accord with Eq. (3.4). At time $t=O(20)$, the ionization is strong enough that field saturation sets in and two asymmetrically propagating fronts emerge. The one propagating 

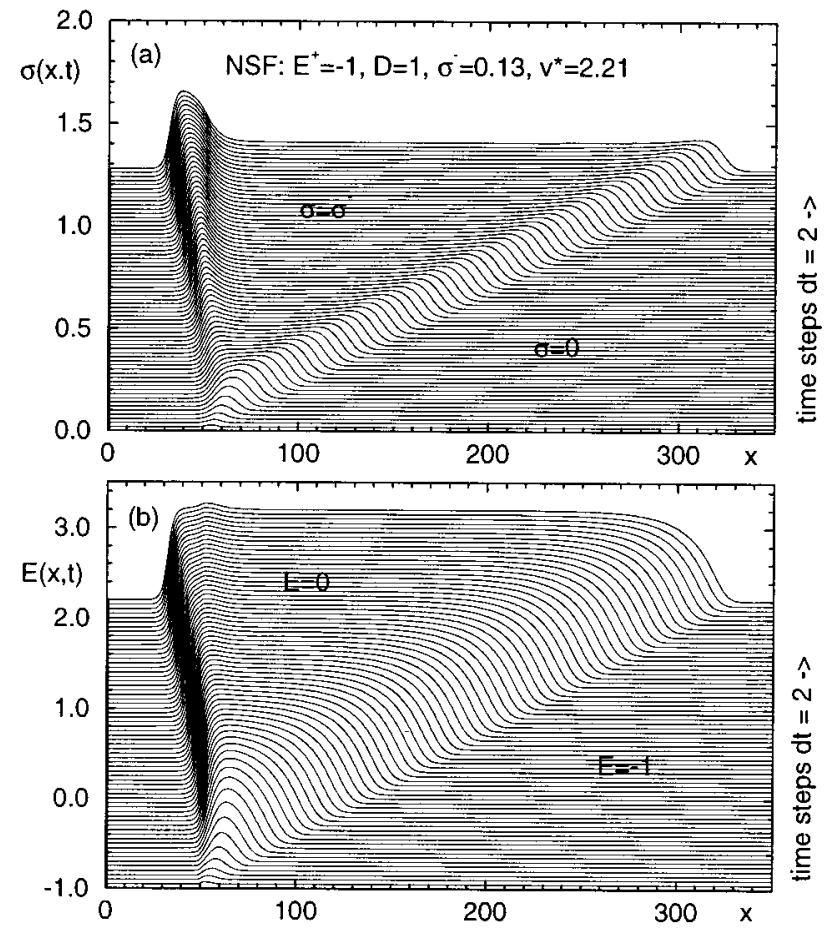

FIG. 7. Numerical integration of the time evolution given by Eqs. (3.10) and (3.11) for $D=1.0$ in a constant background field $E=-1$ (numerical grid size $\Delta x=0.1$ and time step $\Delta \tau=0.05$, initial perturbation at $x_{0}=50$ ). Initial condition at $t=0$ : small chargeneutral, ionized region of Gaussian shape depicted by the lowest line. Each new line corresponds to a time step $\Delta t=2$ and the upper line to $t=130$. (a) The electron density $\sigma(x, t)$ initially grows and then, after field screening in the middle sets in, develops into a NSF propagating to the right and a PSF propagating to the left. (b) The electric field $E(x, t)$ stays $E=-1$ in the nonionized region and becomes dynamically screened to zero in the ionized region.

to the right develops into a uniformly propagating NSF with velocity $v^{*}=2.21$ [53] and degree of ionization behind the front $\sigma^{-}=0.130$. The maximum value of $\sigma$ in the rear part of the front is $\sigma_{\max }=0.150$. At the same time, a structure

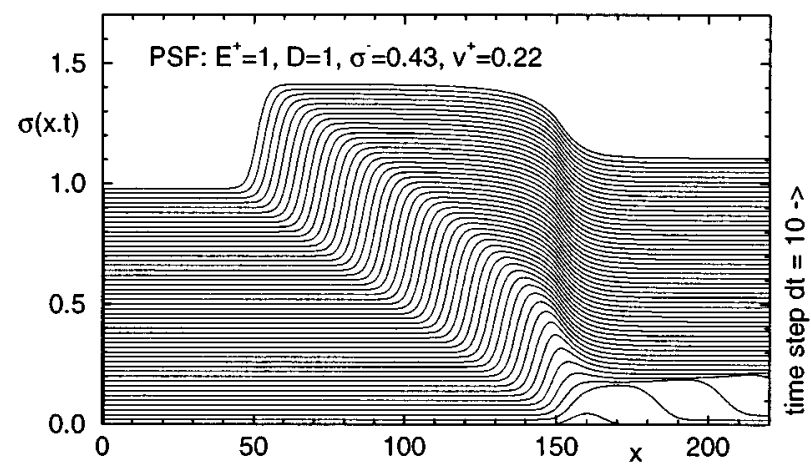

FIG. 8. Emergence of the uniformly translating PSF on the left in the system of Fig. 7. Conditions identical to Fig. 7 except for $x_{0}=150$ and different numerical grid size $(\Delta x=0.05$ and $\Delta \tau=0.01) . \sigma(x, t)$ is shown in the time range $t=0-500$ in time steps $\Delta t=10$. Note the difference in the duration of the transient regimes, in the propagation velocities of PSF and NSF, and in the degrees of ionization behind these.

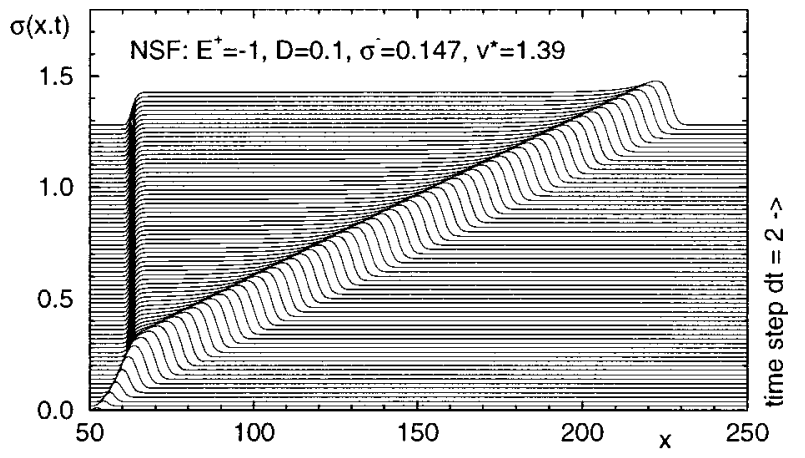

FIG. 9. Identical with Fig. 7(a), except that here $D=0.1$. Time range also $t=0-130$ in steps of $\Delta t=2$. The NSF has sharper contours and propagates slower than for $D=1$, the PSF appears not to develop.

develops on the left, which at time $t=130$ has not yet reached a stationary form, and which eventually will develop into a PSF. (Note that propagation to the left into a negative field $-E^{+}$corresponds to a PSF front moving to the right towards $x \rightarrow \infty$ in a field $+E^{+}$.) How the PSF actually reaches a uniformly translating profile is shown in Fig. 8, where the development for $x_{0}=150$ and otherwise identical initial and boundary conditions is followed in time steps of $\Delta t=10$ during the time $t=0-500$. An asymptotic velocity of $v^{\dagger}=0.22$ and a degree of ionization $\sigma^{-}=0.43$ is reached. Note the huge difference in the degree of ionization and in the front velocity already for the unrealistically large value of the diffusion constant $D=1$.

The predictions from Sec. $\mathrm{V}$ for the selected uniformly translating fronts for $D=1$ and $E^{+}= \pm 1$ yield for the NSF $v^{*}=2.213$ and $\sigma^{-}=0.129$, and for the PSF $v^{\dagger}=0.2199$ and $\sigma^{-}=0.432$. They thus correctly predict the simulations of the initial value problem shown in Figs. 7 and 8 within the accuracy given. Note that for the velocity $v^{\dagger}$ of the PSF and for the degrees of ionization $\sigma^{-}$both behind the PSF and the NSF, this fact also shows the relative accuracy of the two very different numerical methods used, while for the velocity $v^{*}$ of the NSF the numerical integration of the initial value problem exactly reproduces the analytic result.

As $D$ decreases, both the structures within the fronts and the asymmetry between NSF and PSF become more pro-

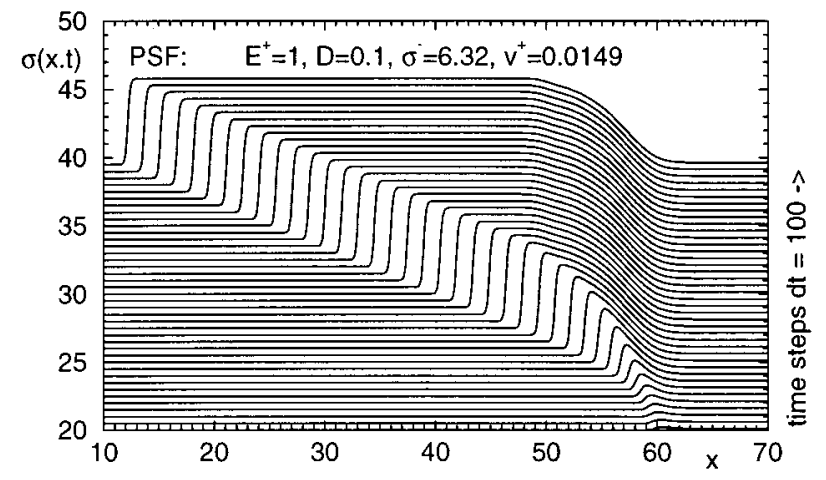

FIG. 10. Emergence of the uniformly translating PSF on the left for $D=0.1$. Initial conditions identical with Fig. 9. The time range $t=4000-8000$ after an initial perturbation at $t=0$ and $x_{0}=60$ is shown in time steps of $\Delta t=100$. (Numerical grid size $\Delta x=0.01$ and $\Delta \tau=0.5$.) 


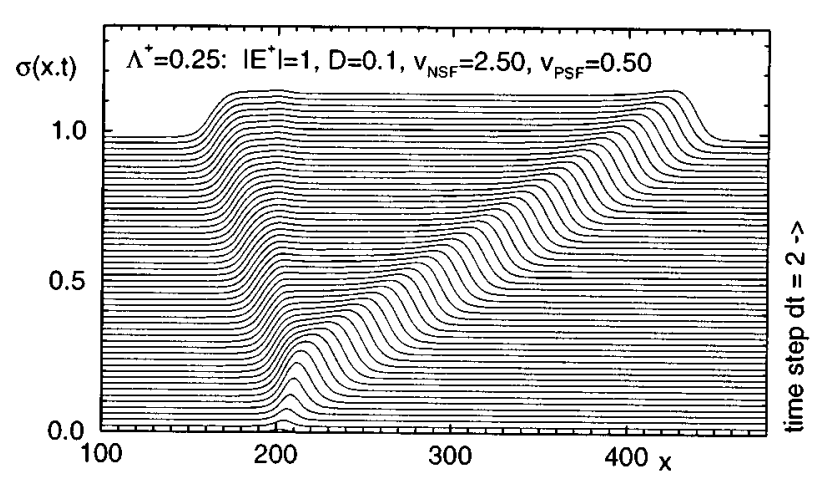

FIG. 11. A nonlocalized initial condition with $\Lambda=0.25$ as described in the text; otherwise, the situation is like in Fig. 9, and $D=0.1$.

nounced. We illustrate this in Figs. 9 and 10 with the temporal development starting from the same initial perturbation as before, but now for $D=0.1$, the value corresponding to the parameter values of the earlier three-dimensional simulations $[8,9]$. The time ranges in each plot are chosen appropriately for seeing the NSF and the PSF evolve into a uniformly translating state. Figure 9 shows a perturbation (initially localized at $x_{0}=50$ ) evolving during time $t=0$ 130 in time steps $\Delta t=2$. Except for the smaller diffusion constant and the stretched $x$ axis, the situation is thus identical with that of Fig. 7. The NSF on the right propagates with a somewhat smaller velocity $v^{*}=1.39$, leaving a slightly higher ionization $\sigma^{-}=0.147$ behind. The maximum $\sigma_{\max }=0.199$ is relatively higher, since diffusional smoothening of structures is less pronounced. On the time scales of Fig. 9, the left front does not propagate, but retracts into an apparently immobile structure. The electrons drift with the field into the ionized region, leaving a layer of screening ions behind. Thus the electrons and the field are almost separated such that ionization on this side almost cannot occur. Eventually few electrons will reach the nonzero field region by diffusion and slowly will build up a higher ionization and ultimately a propagating PSF. That a PSF actually emerges is shown in Fig. 10. Only times $t=4000-8000$ in time steps of $\Delta t=100$ after the initial perturbation at $t=0$ and $x_{0}=60$ are shown. The front propagates with velocity $v^{\dagger}=0.0149$ leaving behind an ionization $\sigma^{-}=6.32$. The numerical values predicted in Sec. V are $v^{*}=1.384$ and $\sigma^{-}=0.144$ for NSF, and $v^{\dagger}=0.0146$ and $\sigma^{-}=6.234$ for PSF. The remaining numerical discrepancy of maximally $2 \%$ could be resolved by choosing a still smaller grid size in Figs. 9 and 10. Comparison of the PSF for $D=1$ and $D=0.1$ indicates that the time it takes such a front to build up rapidly increases with decreasing $D$, but we have not pursued the scaling of the transient time with $D$.

We finally show in Fig. 11 the evolution of streamer fronts starting from nonlocalized initial conditions, i.e., not obeying the bounds (5.5) or (5.6) for $D=0.1$. We used an initial electron density profile $\sigma(x, t=0)$ $=0.01 /(2 \cosh \Lambda(x-200))$ with $\Lambda=0.25$ and an initial field $E=-1$. At these values, for the NSF, $\Lambda_{-}^{+}\left(v^{*}\right)=1.918$ and for the PSF, $\Lambda_{-}^{+}\left(v^{\dagger}\right)=0.3766$. In this case, the bounds (5.5) or (5.6) are indeed violated for both fronts, and Eq. (5.7) predicts a PSF with velocity $v=0.497>v^{\dagger}=0.0146$ and an
NSF with velocity $v=2.497>v^{*}=1.384$. The simulations find the fronts propagating with velocities 0.50 and 2.50 , respectively. The ionization behind the NSF is $\sigma^{-}=0.149$ and behind the PSF $\sigma^{-}=0.158$, so that now both are comparable to each other and to $\sigma^{-}(D=0)=0.1485$ found from Fig. 5(a). Note that the diffusion constant is identical with that of Figs. 9 and 10, the only difference being the extended initial perturbation.

The simulations confirm that streamer front propagation is indeed correctly described by the marginal stability scenario, which in the present case amounts to the statement that the slowest physical velocity is selected, whenever one starts from sufficiently localized initial conditions, just as for the simpler case (5.1).

\section{CONCLUSIONS AND OUTLOOK}

The analysis in this paper fully supports the validity of an effective interface approach suggested by the results of the full three-dimensional simulations of Dhali and Williams and of Vitello et al. [8,9]. This emerges from our detailed study of the associated one-dimensional problem, which yields the following results.

(a) After a very brief stage of transient exponential amplification of the initial ionized seed, the growing streamer evolves into an electrically screened plasma body separating two narrow fronts which propagate into the nonionized outer region. We show that these two fronts correspond, for all practical purposes, to translating profiles which propagate independently. This entails that the separation of spatial scales between an inner front and an outer one, set by the global geometry, is indeed justified.

(b) This enables us to draw upon the existing knowledge about front propagation into unstable states and thus to provide definite predictions about (i) the relationship $v_{f}\left(E^{+}\right)$ between the velocity of a planar streamer front and the value of the electric field ahead of it, and (ii) the value of the degree of ionization of the plasma created by the front, $\sigma^{-}\left(E^{+}\right)$. These predictions, although only valid as such in the absence of front curvature, still compare very favorably with the numerical results of Ref. [9]. The two values of $\sigma^{-}\left(E^{+}\right)$on the axis of Figs. 1(a) and 1(b) behind the curved fronts of the 3D simulations [9] (with the convention that $E^{+}$should be understood as the electric field value extrapolated from the external nonionized region to the front position) are plotted in Fig. 5(a). Without adjustable parameters our one-dimensional predictions for $\sigma^{-}\left(E^{+}\right)$are well within a factor of 2 from the 3D simulations. Likewise, the velocity values for $v_{f}\left(E^{+}\right)$even agree to about $20 \%$.

Moreover, our analysis shows that NSF and PSF propagate in this model and for realistic values of the reduced diffusion coefficient $D$, in a very asymmetric manner.

(i) NSF rapidly reach a regime of uniform propagation typically on the scale of several tens of time units, i.e., in less than $10^{-10} \mathrm{~s}$. Their velocity is slightly larger than the electron drift velocity in the field $E^{+}$.

(ii) This is to be contrasted with the dynamics of PSF: For realistic $D$ values, of order $0.1-0.3$, they approach uniform translation considerably more slowly than NSF - typically on the time scale of $10^{-8} \mathrm{~s}$. Moreover, their asymptotic velocity is also much smaller than $v_{f}^{\mathrm{NSF}}$. It obeys the inequality 
$v_{f}^{\mathrm{PSF}}<D E^{+} \exp \left(-1 / E^{+}\right)$[47]. Finally, while the widths of PSF and NSF are comparable, the degree of ionization behind PSF is much larger (up to a hundred times for $D=0.1$ ) than that behind NSF.

These results answer the question of whether PSF do or do not propagate, while explaining why the simulations of Vitello et al. [9] could not yield a definite answer - most probably because, although their total width is of order $\alpha_{0}^{-1}$, their true inner length scale, as defined by the steepness of the profile, was too small to be resolved by their grid size. [Note that the apparent symmetry between PSF and NSF found in earlier simulations [8] is to be related to the fact that their propagation into a preionized medium (with initial electron density of $10^{8} / \mathrm{cm}^{3}$ ) is studied, and possibly also due to the use of a poorly resolving grid.]

It was observed empirically by Dhali and Williams [8] that in the three-dimensional simulations, the dielectric relaxation time in the plasma behind the front was of the same order as the intrinsic time scale set by the front motion. Our analysis shows that this was no accident: It is a manifestation of the fact that the balance of the growth mechanism (impact ionization) and the stabilization mechanism (screening) leads to a single time scale $t_{0}=\left(\alpha_{0} \mu_{e} E_{0}\right)^{-1}$ for a NSF and for the relaxation behind it for fields of order $E_{0}$. Since our dimensionless value of $\sigma^{-}$is the inverse dielectric relaxation time, it is of order unity (or slightly smaller) for fields $E^{+} \approx-1$.

Of course, the above results should only be considered as a first step towards a realistic treatment of streamer propagation. They will have to be developed and extended along two different directions, as follows.

(i) Predictions of patterns within the present model and comparison with the simulations. Within the frame of the present continuous and fully deterministic model, here we have only considered the restricted case of a one-dimensional geometry. This enabled us to demonstrate that the concept of effective interfaces does apply to streamers. This approach will now have to be extended to the description of curved fronts. As also discussed in [19], one will then be equipped with a reduced formulation, valid on the outer scale, which will permit us to study real three-dimensional streamers as pattern-forming systems, as was done, e.g., for viscous fingers and dendritic solidification fronts [20]. This should provide a direct approach to the question of dielectric patterns, alternative to the phenomenological DLA-inspired dielectric breakdown models [54].

(ii) Possibly, extensions of the model will be necessary to predict real experiments. We have based our analysis on the minimal model as defined in Sec. II. It contains several restricting simplifications. A first step in the improvement of the model would be to include the field dependence of the transport coefficients $D_{e}$ and $\mu_{e}$. It is clear that this will not modify our qualitative analysis, as, e.g., the counting argument for the existence of front solutions in Sec. IV depends only on the linearization about the stable and unstable states. Moreover, the qualitative asymmetry between the NSF and PSF will persist as these result from the asymmetry of the electron drift. Quantitatively, the value of $v^{*}$, the selected value of NSF, will simply be given by Eq. (5.3) with the transport coefficient and ionization rate evaluated at the field value $E^{+}$. The slow transient build-up and small speed of PSF could be affected quantitatively by ionic motion, but from this effect, we expect no major qualitative differences.

Finally, it should be kept in mind that our continuum equations are only valid on length scales larger than the mean free path $l_{\mathrm{mfp}}$. On the other hand, we find for the strongest field values appearing in the simulations (which are much larger than the values of the field across the gap, due to the enhancement near the streamer tip), that the front width decreases down to about $\alpha_{0}^{-1} \approx 3 l_{\mathrm{mfp}}$ in the approximation (2.7). In such limits, nonlocality of the transport and ionization effects begin to play a role. In addition, under these conditions, a typical volume of size $l_{\mathrm{mfp}}^{3}$ contains only of the order of 1000 electrons for the parameter values (2.8) used in the simulations. Fluctuations are then likely to become nonnegligible. In principle, treating these effects calls for a full kinetic description. This is probably out of reach for the moment, but one might want to mimic the main features of these effects by introducing stochastic terms in the equations. These also could mimic photoionization somewhat before the front due to photons released in the impact ionization events, or the natural homogeneous background ionization due to radioactivity and cosmic radiation. Investigation of their relevance for branching of dielectric breakdown patterns might help to understand the asymmetry between the macroscopic patterns of discharges propagating into a positive or a negative field [55].

In conclusion, our analysis opens the way to a microscopically based interface approach to discharges that seems promising for building a coherent framework for the analysis of breakdown patterns of various degrees of complexity.

\section{ACKNOWLEDGMENTS}

W.v.S. gratefully acknowledges hospitality of the Université Paris VII, where this work was initiated. U.E. thanks F. Döbele and A. Stampa for valuable discussions about streamer experiments and plasma physics. Her work was made possible by the Priority Program Non-Linear Systems of the Dutch Science Foundation NWO. We also gratefully acknowledge financial support by NWO and the Lorentz Fund for visits of U.E. and W.v.S. to the Universite Paris VII. Finally, we would like to thank P.A. Vitello for making copies of figures from [9] available, which appear here as Fig. 1.

\section{APPENDIX DIFFERENCES AND SIMILARITIES BETWEEN COMBUSTION AND STREAMER FRONTS}

In the Introduction, we draw on the similarity between the streamer problem and other problems such as combustion, chemical waves, thermal plumes, phase field models, etc., to motivate the development of an effective interface approach. Of these problems, streamer propagation is most closely analogous to combustion, in that the strong nonlinearity of the reaction rates (the combustion rate and the ionization rate) is an important factor in giving rise to front development in flames and streamers, respectively. There are important differences as well, however, and since several interface techniques were originally developed in the context of combustion $[11,12,17]$, we highlight some of the differences and similarities here.

(a) In combustion the reaction rate depends strongly on 
the temperature, whose outer dynamics is governed by a diffusion equation of the form $\partial_{t} T=\nabla^{2} T$, while for streamers the ionization rate depends strongly on the field $|\mathbf{E}|$, with $\mathbf{E}$ the gradient of the potential $\Phi$ that obeys the Laplace equation $\nabla^{2} \Phi \approx 0$ in the outer region where the total charge density vanishes. This field strength $\mathbf{E}$ varies strongly in the streamer front, since the increased screening resulting from the rising electron density suppresses $\mathbf{E}$ - and hence the ionization rate - to zero. In combustion, on the other hand, the temperature hardly varies throughout the combustion zone.

(b) Combustion fronts are essentially fronts progating into a metastable state, because the front has to supply the heat that increases the temperature and hence the reaction rate, while streamer front propagation is an example of front propagation into unstable states, where the reaction starts for any nonvanishing electron density.

(c) In a flame front typically the temperature remains high enough that all the reactions proceed to saturation: all the combustable material burns. The temperature difference between the flame front and the background is then essentially determined by conservation (conversion) of energy. In typical streamer fronts, on the other hand, the field $\mathbf{E}$ is suppressed long before saturation effects start to play a role, and hence the ionization level behind the front is set by the in- ternal dynamics of the front rather than by conservation laws (i.e., the gas density).

(d) The electron drift $-\mu_{e} \mathbf{E}$ has no clear analog in combustion.

(e) Finally, the relevant asymptotic expansion for streamers is not quite like the "activation energy asymptotics" of combustion $[11,12]$, since we consider here fields strengths that are comparable to the characteristic field scale $E_{0}$ of the ionization rate given in Eq. (2.6) before the front, whereas in combustion activation energy asymptotics is often appropriate since the flame temperature remains much smaller than the chemical activation energy. For streamers, an analysis like activation energy asymptotics is appropriate in the limit of small fields $|\mathbf{E}| \ll E_{0}$. Of course, in streamers the rapid variation of the field $\mathbf{E}$ in the front region, and hence the rapid suppression of the ionization rate, looks, at first sight, similar to the suppression of the chemical reaction rate with decreasing temperature in flames. However, in flames this is due to the strongly nonlinear dependence of the reaction rate on temperature before the front (so that a slight suppression of the temperature reduces the reaction rate dramatically), while in streamers in large external fields of order $E_{0}$ this is due to the fact that the field itself is reduced significantly behind the streamer front, as a result of screening.
[1] I. Gallimberti, J. Phys. (France) Colloq. C7-40, 193 (1979).

[2] A. Bondiou and I. Gallimberti, J. Phys. D. 271252 (1994).

[3] Y.P. Raizer, Gas Discharge Physics (Springer, Berlin, 1991).

[4] A.N. Lagarkov and I.M. Rutkevich, Ionization Waves in Electrical Breakdown of Gases (Springer, New York, 1994).

[5] L.M. Vasilyak, S.V. Kostyuchenko, N.N. Kudryavtsev, and I.V. Filyugin, Usp. Fiz. Nauk. 164, 263 (1994) [Phys. Usp. 37, 247 (1994)].

[6] The distinction between the various discharge phenomena seems to vary between authors.

[7] See, e.g., D. Braun, U. Küchler, and G. Pietsch, J. Phys. D 24, 564 (1991); W.R. Rutgers, in: Proceedings of the XXI International Conference on Phenomena in Ionized Gases, edited by G. Ecker, U. Arendt, and J. Böseler (RUB, Bochum, 1993); U. Kogelschatz, in Proceedings of the X International Conference on Gas Discharges and their Applications, edited by W.T. Williams (Univ. College Swansea, Swansea, 1992), Vol. 2, p 972.

[8] S.K. Dhali and P.F. Williams, Phys. Rev. A 31, 1219 (1985); J. Appl. Phys. 62, 4696 (1987).

[9] P.A. Vitello, B.M. Penetrante, and J.N. Bardsley, Phys. Rev. E 49, 5574 (1994). See also P.A. Vitello, B.M. Penetrante, and J.N. Bardsley, in Non-Thermal Plasma Techniques for Pollution Control, edited by B.M. Penetrante and S.E. Schultheis (Springer, Heidelberg, 1993).

[10] M C. Wang and E.E. Kunhardt, Phys. Rev. A 42, 2366 (1990). See references in this article to earlier numerical studies by Kunhardt and co-workers.

[11] F.A. Williams, Combustion Theory (Benjamin/Cummings, Menlo Park, 1985).

[12] J.D. Buckmaster and G.S.S. Ludford, Theory of Laminar Flames (Cambridge University Press, Cambridge, 1982), or
J.D. Buckmaster and G.S.S. Ludford, Lectures on Mathematical Combustion (SIAM, Philadelphia, 1983); a nice elementary account of several of the essential ingredients of flame fronts and their instabilities can be found in J.D. Buckmaster, Physica 12D, 173 (1984).

[13] M. van Dyke, Perturbation Methods in Fluid Dynamics (Parabolic, Stanford, 1975).

[14] C.M. Bender and S.A. Orszag, Advanced Mathematical Methods for Scientists and Engineers (McGraw-Hill, New York, 1978).

[15] E. Meron, Phys. Rep. 218, 1 (1992).

[16] G. Zocchi, P. Tabeling, and M. Ben-Amar, Phys. Rev. Lett. 69, 601 (1992); M. Ben-Amar, Phys. Fluids A 4, 2641 (1992).

[17] P. Fife, Dynamics of Internal Layers and Diffusive Interfaces (SIAM, Philadelphia, 1988).

[18] A. Karma and W.J. Rappel, Phys. Rev. E 53, R3107 (1996), and references therein.

[19] U. Ebert, W. van Saarloos, and C. Caroli, Phys. Rev. Lett. 77, 4178 (1996).

[20] Some important reviews of recent advances are J.S. Langer, in Chance and Matter, edited by J. Souletie (North-Holland, Amsterdam, 1987); D.A. Kessler, J. Koplik, and H. Levine, Adv. Phys. 37, 255 (1988); P. Pelcé, Dynamics of Curved Fronts (Academic, Boston, 1988); and Y. Pomeau and M. Ben Amar, in Solids far from Equilibrium, edited by C. Godreche (Cambridge University Press, Cambridge, 1992).

[21] E. Ben-Jacob, H.R. Brandt, G. Dee, L. Kramer, and J.S. Langer, Physica 14D, 348 (1985).

[22] W. van Saarloos, Phys. Rev. A 37, 211 (1988).

[23] W. van Saarloos, Phys. Rev. A 39, 6367 (1989).

[24] W. van Saarloos and P.C. Hohenberg, Physica D 56, 303 (1992); 69, 209(E) (1993). 
[25] G.C. Paquette and Y. Oono, Phys. Rev. E 49, 2368 (1994); G.C. Paquette, L.-Y. Chen, N. Goldenfeld, and Y. Oono, Phys. Rev. Lett. 72, 76 (1994).

[26] D.L. Turcotte and R.S.B. Ong, J. Plasma Phys. 2, 145 (1968).

[27] N.W. Albright and D.A. Tidman, Phys. Fluids 15, 86 (1972).

[28] R.G. Fowler, Adv. Electron. Electron Phys. 35, 1 (1974); 41, 1 (1976).

[29] M.I. D’yakonov and V.Y. Kachorovskii, Zh. Éksp. Teor. Fiz. 95, 1850 (1989) [Sov. Phys. JETP 68, 1070 (1989)]. We have been informed (V. Shumeiko, private communication with W. Van Saarloos), that D'yakonov has also pointed out in a talk the strong similarity between streamers and other problems of pattern formation such as dendrites.

[30] Instead of distinguishing the two different types of streamers with the acronyms ADS (anode directed streamer) and CDS (cathode directed streamer) as is sometimes done, we prefer to use NSF (negative streamer front) and PSF (positive streamer front), as this wording is more appropriate for an effective interface approach.

[31] D.G. Aronson and H.F. Weinberger, in Partial Differential Equations and Related Topics, edited by J.A. Goldstein (Springer, Heidelberg, 1975); Adv. Math. 30, 33 (1978).

[32] P. Collet and J.-P. Eckmann, Instabilities and Fronts in Extended Systems (Princeton University Press, Princeton, 1990).

[33] A. Kolmogorov, I. Petrovsky, and N. Piskunov, Bull. Univ. Moskou, Ser. Internat., Sec. A 1, 1 (1937).

[34] R.A. Fisher, Ann. Eugenics 7, 355 (1937).

[35] A short calculation shows that a homogeneous current $|\mathbf{j}|=1$ generates magnetic fields, whose Lorentz force on the free charges moving with the drift velocity $\mu_{e} E_{0}$ becomes comparable to the force of an electric field of order $E_{0}$, when the current extends over a region of width $l_{B}$ $=\alpha_{0}^{-1} c^{2} /\left(\mu_{e} E_{0}\right)^{2}=1.6 \times 10^{5} \alpha_{0}^{-1} \approx 0.4 \mathrm{~m}$ for the parameters of $[8,9]$. In practice, streamer dimensions are much smaller than $l_{B}$, and hence the magnetic fields can generally be neglected.

[36] S.K. Dhali and A.P. Pal, J. Appl. Phys. 63, 1355 (1988).

[37] It is useful to realize that our model is inaccurate for small fields, since any nonzero electron density is unstable in our model for any field strength, due in particular to the neglect of recombination terms. A similar situation occurs in combustion; there, the exponential reaction rate is sometimes modified by introducing a so-called switch-on temperature, below which the combustion rate is zero. As discussed in [12], the results do not strongly depend on the switch-on temperature provided it is low enough, and an asymptotic expansion in fact nicely circumvents the introduction of this temperature. Similar observations hold for the streamer problem: A crude way to circumvent the introduction of recombination terms would be to introduce a threshold field $E_{\text {thr }}$ below which $f(|\mathbf{E}|)$ vanishes. Essentially all of our analysis applies to such cases as well, and the results do not depend sensitively on the value of this field.

[38] One should be aware that it is possible that the state generated by the front is actually unstable or metastable. In such cases the front is usually followed by a second front invading the unstable or metastable state. See, e.g., K. Nozaki and N. Bekki, Phys. Rev. Lett. 51, 2171 (1983), and F.J. Elmer, J. Burns, and H. Suhl, Europhys. Lett. 22399 (1993).

[39] The discussion is easily generalized to the case in which the ionization function $f(|\mathbf{E}|)=0$ in an interval $E \in\left[0, E_{\mathrm{thr}}\right]-$ compare Ref. [37]. In this case, ionization is absent for any field $E<E_{\mathrm{thr}}$, so that there is a larger multiplicity of stationary states of the model, and so that for fields below this threshold the dynamics is due to conventional electrodynamics with conserved particle densities. This generalization, however, does not affect our conclusions about front propagation qualitatively: It is easy to convince oneself that the state generated after long times by a propagating front is still the one with $\mathbf{E}=\mathbf{0}$, since as long as the field behind the front is nonzero but of the same sign as $E^{+}$, charge is pumped into the streamer head from behind in such a way that the field jump across the streamer head increases in time. Since $E^{+}$is fixed, this implies that the field behind the streamer head decays.

[40] E.D. Lozanskii, Zh. Tekh. Fiz. 38, 1563 (1968) [Sov. Phys. Tech. Phys. 13, 1269 (1969)].

[41] W.K.H. Panofsky and M. Phillips, Classical Electricity and Magnetism (Addison-Wesley, Reading, MA, 1962), p. 122.

[42] This is argued in Appendix A of [22]: For equations like ours that admit uniformly translating front solutions, one generally expects a one-parameter family of front solutions, a feature well known for the case of the nonlinear diffusion equation discussed briefly in Sec. V. For pattern forming fronts propagating into an unstable state, one typically has a two parameter family of fronts - see, e.g., [24] or P. Collet and J.-P. Eckmann, Commun. Math. Phys. 107, 39 (1986), although a general proof is lacking.

[43] M. Abramowitz and I.A. Stegun, Handbook of Mathematical Functions (Dover, New York, 1972).

[44] W.H. Press, B.R. Flannery, S.A. Teukolsky, and W.T. Vetterling, Numerical Recipes (Cambridge University Press, Cambridge, 1986).

[45] Of course, in principle, in a three-dimensional dynamical system one can have limit cycles and other (strange) attractors. Limit cycles would in our case correspond to spatially periodic ionization waves, but the occurrence of such solutions is clearly excluded physically by the fact that the ion density must be a monotonically increasing function of $\xi$. Other types of nontrivial behavior of the flow are excluded for the same reason.

[46] See, e.g., R.D. Benguria and M.C. Depassier, Phys. Rev. Lett. 73, 2272 (1994); A. Goriely, ibid. 75, 2047 (1995).

[47] U. Ebert (unpublished).

[48] I.M. Gel'fand, Usp. Mat. Nauk 14, No. 2 (86) 87 (1959) [Am. Math. Soc. Transl. Ser. 2 29, 295 (1963)].

[49] G.T. Dee and J.S. Langer, Phys. Rev. Lett. 50, 383 (1983).

[50] G.T. Dee and W. van Saarloos, Phys. Rev. Lett. 60, 264 (1988).

[51] For a review, see A. Bers, in Handbook of Plasma Physics, edited by M.N. Rosenbluth and R.Z. Sagdeev (North-Holland, Amsterdam, 1983), Vol. 1.

[52] E.M. Lifshitz and L.P. Pitaevskii, Physical Kinetics, Course of Theoretical Physics Vol. 10 (Pergamon, New York, 1981), Chap. VI.

[53] By tracking the position $x_{f}(\tau)$ of the point where $\sigma\left(x_{f}(\tau), \tau\right)=0.1 \sigma^{-}$, we followed the convergence of the local velocity $v_{f}(\tau)=d x(\tau) / d \tau$ towards $v^{*}$, and found it to be consistent with the formula $v_{f}(\tau)=v^{*}-1.5 D /\left(\sqrt{f\left|E^{+}\right|} \tau\right)+\cdots$ derived in [23].

[54] L. Niemeyer, L. Pietronero, and H.J. Wiessmann, Phys. Rev. Lett. 52, 1033 (1984).

[55] J.C. Maan, B. Willing, and P. Uhlig, Physica B 204, 311 (1995). 